\title{
Mechanism of Pacemaker Activity in Zebrafish DC2/4 Dopaminergic Neurons
}

\author{
${ }^{\circledR}$ Vladimir A. Ilin, ${ }^{1,2}$ Qing Bai, ${ }^{1,2}$ Alan M. Watson, ${ }^{3}{ }^{\circledR}$ Maxim Volgushev, ${ }^{4}$ and ${ }^{\circledR}$ Edward A. Burton ${ }^{1,2,5,6}$ \\ ${ }^{1}$ Department of Neurology, University of Pittsburgh, Pittsburgh, Pennsylvania 15260, ${ }^{2}$ Pittsburgh Institute for Neurodegenerative Diseases, \\ University of Pittsburgh, Pittsburgh, Pennsylvania 15213, ${ }^{3}$ Center for Biologic Imaging, University of Pittsburgh, Pittsburgh, Pennsylvania 15261, \\ ${ }^{4}$ Department of Psychological Sciences, University of Connecticut, Storrs, Connecticut 06269, ${ }^{5}$ Department of Microbiology and Molecular Genetics, \\ University of Pittsburgh, Pittsburgh, Pennsylvania 15260, and ${ }^{6}$ Geriatric Research, Education and Clinical Center, Pittsburgh VA Healthcare System, \\ Pittsburgh, Pennsylvania 15240
}

Zebrafish models are used increasingly to study the molecular pathogenesis of Parkinson's disease (PD), owing to the extensive array of techniques available for their experimental manipulation and analysis. The ascending dopaminergic projection from the posterior tuberculum (TPp; diencephalic populations DC2 and DC4) to the subpallium is considered the zebrafish correlate of the mammalian nigrostriatal projection, but little is known about the neurophysiology of zebrafish DC2/4 neurons. This is an important knowledge gap, because autonomous activity in mammalian substantia nigra ( $\mathrm{SNc}$ ) dopaminergic neurons contributes to their vulnerability in PD models. Using a new transgenic zebrafish line to label living dopaminergic neurons, and a novel brain slice preparation, we conducted whole-cell patch clamp recordings of DC2/4 neurons from adult zebrafish of both sexes. Zebrafish DC2/4 neurons share many physiological properties with mammalian dopaminergic neurons, including the cell-autonomous generation of action potentials. However, in contrast to mammalian dopaminergic neurons, the pacemaker driving intrinsic rhythmic activity in zebrafish DC2/4 neurons does not involve calcium conductances, hyperpolarization-activated cyclic nucleotide-gated (HCN) channels, or sodium leak currents. Instead, voltage clamp recordings and computational models show that interactions between three components - a small, predominantly potassium, leak conductance, voltage-gated sodium channels, and voltage-gated potassium channels - are sufficient for pacemaker activity in zebrafish DC2/4 neurons. These results contribute to understanding the comparative physiology of the dopaminergic system and provide a conceptual basis for interpreting data derived from zebrafish PD models. The findings further suggest new experimental opportunities to address the role of dopaminergic pacemaker activity in the pathogenesis of PD.

Key words: computational; dopamine; electrophysiology; pacemaker; Parkinson's disease; zebrafish

Significance Statement

Posterior tuberculum (TPp) DC2/4 dopaminergic neurons are considered the zebrafish correlate of mammalian substantia nigra (SNc) neurons, whose degeneration causes the motor signs of Parkinson's disease (PD). Our study shows that DC2/4 and SNc neurons share a number of electrophysiological properties, including depolarized membrane potential, high input resistance, and continual, cell-autonomous pacemaker activity, that strengthen the basis for the increasing use of zebrafish models to study the molecular pathogenesis of PD. The mechanisms driving pacemaker activity differ between DC2/4 and SNc neurons, providing: (1) experimental opportunities to dissociate the contributions of intrinsic activity and underlying pacemaker currents to pathogenesis; and (2) essential information for the design and interpretation of studies using zebrafish PD models.

\footnotetext{
Received Aug. 12, 2020; revised Feb. 2, 2021; accepted Feb. 7, 2021.

Author contributions: V.A.I., A.M.W., M.V., and E.A.B. designed research; V.A.I., Q.B., A.M.W., and E.A.B. performed research; Q.B. and E.A.B. contributed unpublished reagents/analytic tools; V.A.I., M.V., and E.A.B. analyzed data; V.A.I., A.M.W., M.V., and E.A.B. wrote the paper.

This work was supported by the National Institutes of Health (ES022644), by a VAPHS GRECC Pilot Project award, and by the University of Pittsburgh Medical Center (endowed chair to E.A.B.). We thank Prof. E. Aizenman (University of Pittsburgh) for helpful technical advice and discussion and the aquatics team in the Department of Laboratory Animal Resources at the University of Pittsburgh for expert care of our transgenic zebrafish. The contents of this article do not represent the views of the US Government.

V. A. Ilin's present address: UPMC Graduate Medical Education, Pittsburgh, PA 15213.

The authors declare no competing financial interests.

Correspondence should be addressed to Edward A. Burton at eab25@pitt.edu.

https://doi.org/10.1523/JNEUROSCI.2124-20.2021

Copyright $\odot 2021$ the authors
}

\section{Introduction}

The core motor abnormalities of Parkinson's disease (PD), tremor, rigidity, and bradykinesia, are caused by degeneration of substantia nigra (SNc) dopaminergic neurons. Multiple etiologic factors, both genetic and environmental, are thought to converge on a limited number of shared pathophysiological pathways, including abnormalities of mitochondrial function and proteostasis. However, the factors governing selective vulnerability of dopaminergic neurons are not well understood. This is a key question, because elucidating the underlying mechanisms may facilitate the development of treatments that prevent the 
progression of motor symptoms in PD. Several possibilities have been suggested (for review, see Surmeier, 2018). Robust expression of the PD-associated protein $\alpha$-synuclein in SNc dopaminergic neurons (Zharikov et al., 2015) may compromise mitochondrial function (Hsu et al., 2000; Devi et al., 2008; Banerjee et al., 2010; Di Maio et al., 2016) and enhance susceptibility to formation and propagation of protein aggregates (Desplats et al., 2009; Luk et al., 2009). Degradation of cytosolic dopamine generates reactive oxygen species that contribute to oxidative stress (Hastings, 2009) and dopamine is readily oxidized to yield a reactive quinone that can modify cellular proteins (Van Laar et al., 2008; Hauser et al., 2013). The disproportionately long, poorly-myelinated axons of SNc dopaminergic neurons (Braak and Del Tredici, 2004) and multiple neurotransmitter release sites (Matsuda et al., 2009) may enhance cellular demands on mitochondrial metabolism. Finally, metabolic stress may be amplified by continuous autonomous firing of dopaminergic neurons, which discharge action potentials (APs) even in the absence of synaptic inputs (Surmeier, 2018). This continual rhythmic activity is driven by cell-intrinsic pacemaker mechanisms and has been observed in the intact CNS (Grace and Bunney, 1983), brain slice preparations (Grace and Onn, 1989; Yung et al., 1991), and isolated cells (Kim et al., 2007; Puopolo et al., 2007). Pacemaker activity is predicted to augment the bioenergetic demands placed on dopaminergic neuronal mitochondria. Moreover, calcium pacemaker currents in the adult mouse SNc have been shown to contribute to pathology in experimental models of PD (Chan et al., 2007).

Since many of the proposed mechanisms mediating cellular vulnerability in PD relate to intrinsic properties of differentiated dopaminergic neurons in vivo, the pathophysiology underlying neurodegeneration is frequently studied using animal models. Zebrafish models present several advantages for analyzing the molecular and cellular basis for degeneration of dopaminergic neurons, including an extensive array of available experimental approaches such as transgenesis (Wen et al., 2008), reverse genetics (Watson et al., 2019), intravital imaging (Van Laar et al., 2020), chemoptogenetics (Xie et al., 2020), and chemical modifier screening (Zhang et al., 2017). Consequently, zebrafish models have been exploited increasingly to provide insights into PD pathophysiology (for review, see Bartel et al., 2020). There is no nigrostriatal projection in the zebrafish CNS. Ascending dopaminergic inputs to the subpallium in both larval and adult zebrafish originate in large pear-shaped neurons located in the periventricular nucleus of the posterior tuberculum (TPp; diencephalic dopaminergic groups DC2 and DC4; Rink and Wullimann, 2001, 2002; Ma, 2003; Tay et al., 2011; Yamamoto et al., 2011). This ascending dopaminergic projection from TPp to the ventral telencephalon is considered the anatomic and functional correlate of the mammalian nigrostriatal projection (Wullimann, 2014). However, little is known about which factors implicated in the selective vulnerability of dopaminergic neurons in PD are replicated in DC2/4 neurons; in particular, their pacemaker properties have not been studied. Given the potential importance of cell-intrinsic autonomous activity in the degeneration of dopaminergic neurons in $\mathrm{PD}$, and the growing interest in exploiting zebrafish models for elucidating underlying pathogenic mechanisms, this is an important gap in current knowledge.

To elucidate the physiological properties of zebrafish dopaminergic neurons, we developed a brain slice preparation enabling whole cell patch-clamp recordings from these cells. Using a combination of electrophysiological analysis and computer model simulations, we found that zebrafish DC2/4 neurons show robust cell-intrinsic pacemaker activity that is dependent on three essential elements: a weak potassium leak current, voltage-gated sodium channels, and voltage-gated potassium channels.

\section{Materials and Methods}

Ethical approval

All studies were conducted with approval from the University of Pittsburgh Institutional Animal Care and Use Committee, in accordance with the NIH Guide for the Care and Use of Laboratory Animals.

\section{Transgenic zebrafish}

Approximately equal numbers of male and female adult zebrafish were used for these studies. Transgenic $\operatorname{Tg}($ th:gal4-vp16); $\operatorname{Tg}($ UAS:egfp) zebrafish were used to identify dopaminergic neurons in acute brain slices for physiological recordings. Construction and characterization of the novel $\operatorname{Tg}($ th:gal4-vp16) line will be reported in full elsewhere. Briefly, a bacterial artificial chromosome (BAC) clone containing the zebrafish th gene was isolated and an expression cassette for Gal4-VP16-PolyA was inserted in-frame into exon three by homologous recombination. The transgenic $\operatorname{Tg}($ th:gal4- $v$ p16) line was made by microinjection of the BAC construct into single-cell zebrafish embryos using the Tol2 transposon method, and expanded from a single F1 founder. For identification of dopaminergic neurons by epifluorescence microscopy, $\operatorname{Tg}($ th:gal4-vp16) and $\operatorname{Tg}(U A S$ :egf $)$ zebrafish were crossed, so that GFP expression was transactivated by Gal4-VP16 in tyrosine hydroxylase (TH)-expressing dopaminergic neurons. The identity of the dopaminergic neurons used for recordings was verified by immunofluorescence for endogenous TH expression, and by their characteristic neuroanatomical location and morphology.

Immunofluorescence and confocal microscopy

Adult zebrafish brains were dissected, fixed, cryoprotected and sectioned exactly as descried in our previous work (Bai et al., 2014). Axial 12- $\mu \mathrm{m}$ thick sections were labeled using primary antibodies to TH (rabbit polyclonal, Millipore, \#AB152; 1:1000) and GFP (chicken polyclonal, Abcam, catalog \#AB13970; 1:5000), followed by Alexa Fluor 488-anti-chicken and Alexa Fluor 555-anti-rabbit secondary antibodies (Invitrogen). Confocal images were acquired using an Olympus IX-71 confocal microscope with Fluoview software. For neuronal labeling during electrophysiological recording, the pipette was filled with intracellular solution supplemented with biocytin $0.2 \%$. After recording was completed, slices were fixed in $4 \%$ paraformaldehyde in $\mathrm{PBS}$ at $4^{\circ} \mathrm{C}$ for $12 \mathrm{~h}$, washed in PBS, incubated in PBS supplemented with $0.1 \%$ Triton X-100 and $1 \%$ normal goat serum for $1 \mathrm{~h}$, then washed again in PBS. Sections were then incubated with streptavidin conjugated to Alexa Fluor 555 (Invitrogen, \#32355; 1:200 dilution in PBS with 1\% normal goat serum), washed in PBS and mounted for imaging.

\section{Solutions and chemicals}

All chemicals were obtained from Sigma, except for tetrodotoxin (TTX), which was obtained from Fisher Scientific. Solutions with the following compositions were used: standard extracellular solution: $131 \mathrm{~mm} \mathrm{NaCl}$, $2 \mathrm{~mm} \mathrm{KCl}, 1.25 \mathrm{~mm} \mathrm{KH}_{2} \mathrm{PO}_{4}, 20 \mathrm{~mm} \mathrm{NaHCO}, 2 \mathrm{~mm} \mathrm{MgCl} 2,2.5 \mathrm{~mm}$ $\mathrm{CaCl}_{2}, 10$ mm glucose, bubbled with $95 \% \mathrm{O}_{2} / 5 \% \mathrm{CO}_{2}, \mathrm{pH} 7.4$; calciumfree extracellular solution: identical to standard extracellular solution, but without $\mathrm{CaCl}_{2}$; sodium-free extracellular solution: $151 \mathrm{~mm}$ choline chloride, $3.25 \mathrm{~mm} \mathrm{KCl}, 2 \mathrm{~mm} \mathrm{MgCl}$, $2.5 \mathrm{~mm} \mathrm{CaCl}_{2}, 10 \mathrm{~mm}$ HEPES, and $10 \mathrm{~mm}$ glucose, $\mathrm{pH} 7.4$, bubbled with air; potassium gluconate-based intracellular solution: $126 \mathrm{~mm}$ K-Glu, $15 \mathrm{~mm} \mathrm{KCl,} 10 \mathrm{~mm} \mathrm{NaCl}, 10 \mathrm{~mm}$ HEPES, and $2 \mathrm{~mm} \mathrm{MgCl}_{2}, \mathrm{pH} 7.2$; cesium-based intracellular solution: $141 \mathrm{~mm} \mathrm{CsCl}, 10 \mathrm{~mm} \mathrm{NaCl}, 10 \mathrm{~mm}$ HEPES, and $2 \mathrm{~mm} \mathrm{MgCl}_{2}, \mathrm{pH}$ 7.2. The following toxins and chemicals were added to extracellular solution where indicated in the text: TTX for complete $(1 \mu \mathrm{M})$ or partial $(30 \mathrm{nM})$ blockade of voltage-gated sodium channels; tetraethylammonium (TEA; $20 \mathrm{~mm}$ ) and 4-aminopyridine (4-AP; $2 \mathrm{~mm}$ ) to block voltage-gated potassium channels; isradipine ( $9 \mu \mathrm{M})$ to block L-type calcium channels; apamin $(100 \mathrm{~nm})$ to block small conductance calcium-activated potassium (SK) channels; cadmium chloride (50 $\mu \mathrm{M})$ to block all calcium channels; $\mathrm{N}$-ethyl-1,6-dihydro-1,2-dimethyl-6-(methylimino)- $N$-phenyl-4- 
pyrimidinamine hydrochloride (ZD7288; $50 \mu \mathrm{M}$ ) to block hyperpolarization-activated cyclic nucleotide-gated ( $\mathrm{HCN})$-channels.

\section{Brain slice preparation}

A $10 \times 10 \times 5$-mm block of $2 \%$ low point melting agarose was prepared, and grooves of $2-3 \times 5-6 \times 2-3 \mathrm{~mm}$ in depth were cut into one side. The block was cooled and placed on a metal plate resting on ice. Brain slices were prepared from zebrafish of both sexes aged between 60 and $300 \mathrm{~d}$ postfertilization. Animals were euthanized individually in $0.02 \%$ buffered tricaine until all gill movements ceased, then cooled in ice-cold water for $1 \mathrm{~min}$. After transferring to ice-cold, oxygenated extracellular solution, the brain was quickly removed from the skull and transferred into a 35-mm culture dish filled with ice-cold, oxygenated extracellular solution. Two to three brains prepared this way were transferred into the grooves of the agarose block, extracellular solution was removed using a soft paper tissue, and a drop of melted $2 \%$ agarose at $38-40^{\circ} \mathrm{C}$ was used to fix the brain to the block; the small volume of melted agarose relative to the larger cooled block ensured its rapid cooling, so that the brains were not damaged. The agarose block was then fixed to the plate of a vibratome (VT1000, Leica) using cyanoacrylate glue. The cutting chamber of the vibratome was filled with cold, oxygenated extracellular solution. Axial brain slices $(200-\mu \mathrm{m}$ thickness) were cut at a low speed $(0.1$ $\mathrm{mm} / \mathrm{s}$ ) starting from the rostral pole; slices containing the telencephalon and the first slice containing the rostral boundary of the optic tectum were discarded. Anatomical landmarks in the next two slices, which contained the diencephalic areas of interest (see Results), were verified using a dissection microscope. The slices were transferred into an incubation chamber comprising a $35-\mathrm{mm}$ culture dish with mesh-screened bottom, housed inside a $5 \times 5 \times 10$-cm plastic container filled with extracellular solution at $22^{\circ} \mathrm{C}$, bubbled continuously with oxygen-carbon dioxide mixture. Brain slices recovered under these conditions for at least $1 \mathrm{~h}$ before recordings were started.

\section{Electrophysiological recording}

For recording, a brain slice was transferred from the incubation chamber to a 35-mm culture dish with a layer of silicone (Sylgard, Corning) at the bottom, and fixed to the silicone using $25-\mu \mathrm{m}$ in diameter tungsten pins. The slice was continuously superfused with oxygenated extracellular solution ( $2 \mathrm{ml} / \mathrm{min}$ at room temperature) for the reminder of the experiment. The dish was mounted on the stage of a microscope (BX51WI; Olympus) equipped with $4 \times$ air or $40 \times$ water-immersion objectives, infrared-DIC optics, epifluorescence illumination and a video camera (FL3-U3-20E4M-C, Point Gray Research). Borosilicate glass microelectrodes were made using a micropipette puller (P-97; Sutter Instruments) and filled with intracellular solution (resistance 6-10 M 2 ). Electrodes were connected to a head stage (HS-9A x 0.1 or CV203BU; Molecular Devices) mounted on a motorized micromanipulator (MP-225; Sutter). Patch clamp recordings were made in a whole-cell configuration using Axoclamp 900A or Axopatch 200B amplifiers (Molecular Devices). Recorded signals were digitized at a sampling rate of $20 \mathrm{kHz}$ (Digidata 1440A and pCLAMP 10 software; Molecular Devices) and stored on a computer for off-line processing.

After establishing whole-cell recording configuration in voltage clamp mode, the amplifier was switched to current clamp mode and membrane potential traces of 5-s length were recorded every minute throughout the experiment, to evaluate the frequency of autonomous activity and the properties of APs, both at baseline and during application of toxins and chemicals. After recording the baseline for $10-30 \mathrm{~min}$, responses to current injections were measured using 500-ms-long current steps from -300 to $1000 \mathrm{pA}$ in $20-\mathrm{pA}$ increments, injected every $2 \mathrm{~s}$. Responses to negative current steps were used to calculate input resistance; responses to positive steps were used to calculate the frequency response of the pacemaker.

\section{Data analysis}

Off-line analysis was conducted using custom MATLAB applications (MathWorks) and tools from the Clampfit 10.7 module of pCLAMP 10 (Molecular Devices). APs were detected at a threshold of $-10 \mathrm{mV}$. Instantaneous frequency was measured as the reciprocal of the first interspike interval. Adaptation ratio was calculated as the ratio between the last and first interspike intervals in response to a 500-pA current step. Mean frequency was measured as the number of APs divided by the time period during which APs could be detected (in response to a current step, this period could be shorter than step duration because of depolarization block). Membrane potential during interspike intervals was calculated after removing APs $(75 \mathrm{~ms}$ before and after the membrane potential crossed the AP detection threshold) from traces. AP parameters were calculated using averaged AP traces. AP peak and fast after-hyperpolarization (AHP) were measured as the maximum and minimum membrane potentials of the averaged AP, respectively. AP amplitude was measured between AP peak and AHP. AP width was measured at half-amplitude. Slow AHP was defined as the mean of the averaged membrane potential trace between [AP peak +5 (AP width)] and $[\mathrm{AP}$ peak $+75 \mathrm{~ms}]$. AP threshold potential was calculated using a "geometric" approach using the $\mathrm{dV} / \mathrm{dt}$ versus $\mathrm{V}$ phase plot of the averaged AP (Chistiakova et al., 2019). The initial point of the phase plot was connected to the point of maximal $\mathrm{dV} / \mathrm{dt}$ by a straight line and the threshold was defined as the value of the membrane potential $(\mathrm{V})$ at the point of the phase plot located at maximal distance from this line.

\section{Experimental design and statistical analysis}

Statistical analysis was completed using Prism 8 software (GraphPad Software). Paired $t$ tests were used to compare measurements from the same cells before and after an intervention. One-way ANOVA with Tukey's multiple comparisons test was employed to compare three or more groups. Linear regression and Pearson correlation coefficient were employed to determine correlations between parameters. Data describing sample distributions are presented as mean $\pm \mathrm{SD}$, whereas data comparing sample means are shown as mean $\pm \mathrm{SE}$, as indicated in the text. The details of experimental design, including sample sizes and control groups, are indicated in each figure legend.

Measurement of voltage-gated potassium and sodium channel dynamics Potassium current was isolated pharmacologically by adding $1-\mu \mathrm{m}$ TTX to the external medium, and measured in voltage-clamp using responses to 50 -ms-long holding potential steps from $-68 \mathrm{mV}$ to between -63 and $112 \mathrm{mV}$, in 5-mV increments. Current amplitude was measured at the peak of the response. Leak currents were compensated using the P/N leak subtraction protocol of pClamp 10. Peak conductance $G_{K}$ was calculated for each step as:

$$
G_{K}=\frac{I_{K}}{V_{\text {step }}-E_{K}}
$$

where, $I_{K}$ is the peak current response, $V_{\text {step }}$ is the potential of the voltage step, $E_{K}$ is the reversal potential for $\mathrm{K}^{+}$ions $(-96 \mathrm{mV}$; calculated from the Nernst equation). For assessment of potassium channel activation, $G_{K}$ was normalized to its maximum value and plotted against the amplitude of voltage steps. The current responses for each voltage step were fitted to an exponential function (Clampfit 10.7, Molecular Devices):

$$
I(t)=I_{\max }\left(1-e^{-\left(\frac{t}{\tau}\right)}\right)^{4}
$$

where $I(t)$ is amplitude of the current at time $t, I_{\max }$ the maximum amplitude of the current, and $\tau$ the time constant. For assessing the voltage dependence of potassium channel activation, the time constants were plotted against the amplitude of the voltage steps.

Sodium current was isolated pharmacologically by adding $20 \mathrm{~mm}$ TEA and $2 \mathrm{~mm} 4$-AP to the extracellular solution and using a cesiumbased intracellular solution. In order to achieve reliable space clamp, we partially blocked sodium channels with 30 nм TTX and used a voltage prepulse protocol (Milescu et al., 2010). These methods for reducing sodium conductance limit the decrease of the space constant that is associated with activation of sodium channels, thereby making a cell electrotonically more compact and enhancing reliability of space clamp via a somatic electrode (Milescu et al., 2010). In the prepulse protocol, the membrane potential was initially held at $-77 \mathrm{mV}$; and then a $5-\mathrm{ms}$ prepulse to $-32 \mathrm{mV}$ was followed by a $3-\mathrm{ms}$ step to $-52 \mathrm{mV}$, after which 
membrane potential was clamped at values ranging between -47 and $-7 \mathrm{mV}$, in $5-\mathrm{mV}$ increments. To measure sodium current inactivation, $50-\mathrm{ms}$ holding potential steps between -62 to $-12 \mathrm{mV}$ with $5-\mathrm{mV}$ increments were followed by a $20-\mathrm{ms}$ step to $-7 \mathrm{mV}$. Sodium current was measured at the peak of the response. Leak currents were subtracted using the $\mathrm{P} / \mathrm{N}$ leak subtraction protocol of pClamp 10. Peak sodium conductance $G_{N a}$ was calculated as following:

$$
G_{N a}=\frac{I_{N a}}{V_{s t e p}-E_{N a}},
$$

where $I_{\mathrm{Na}}$ is the maximum amplitude of response to $V_{\text {step }}$ and $E_{\mathrm{Na}}$ is the equilibrium potential for $\mathrm{Na}^{+}$ions $(69 \mathrm{mV})$.

To calculate the time constants of activation and inactivation of sodium currents, exponential functions were fitted to the responses (Clampfit 10.7), either from the beginning to the peak $\left[I_{A}(t)\right]$ or from the peak to the end $\left[I_{I}(t)\right]$ :

$$
\begin{aligned}
& I_{A}(t)=I_{\text {max }}\left(1-e^{\left.-\left(\frac{t}{\tau_{A}}\right)\right)^{3}}\right. \\
& I_{I}(t)=I_{\max }\left(1-e^{\left.-\left(\frac{t}{\tau_{I}}\right)\right)},\right.
\end{aligned}
$$

where $I_{\max }$ is maximum amplitude of the current, $\tau_{\mathrm{A}}$ is the activation time constant, and $\tau_{\mathrm{I}}$ is the inactivation time constant. Activation and inactivation time constants were plotted against the amplitudes of the test voltage steps.

The transfer rate functions $\alpha_{\mathrm{n}}(\mathrm{V})$ and $\beta_{\mathrm{n}}(\mathrm{V})$, which determine the activation function $\mathrm{n}(\mathrm{V})$ for potassium channels, were derived using Hodgkin-Huxley formalism (Hodgkin and Huxley, 1952). In Equations 6-8 below, $V$ is membrane potential, $G A_{K}(V)$ is the normalized steady state activation of potassium conductance, and $\tau_{n}(V)$ is an activation time constant for potassium conductance, measured experimentally (see Results). We first calculated $\mathrm{n}(\mathrm{V}), \alpha_{\mathrm{n}}(\mathrm{V})$ and $\beta_{\mathrm{n}}(\mathrm{V})$ from experimental data (discrete potential values measured in recordings).

$$
\begin{gathered}
n(V)=\sqrt[4]{G A_{K}(V)}, \\
\alpha_{n}(V)=\frac{n(V)}{\tau_{n}(V)}, \\
\beta_{n}(V)=\frac{1-n(V)}{\tau_{n}(V)} .
\end{gathered}
$$

Next, we fitted transfer rate functions $\alpha_{\mathrm{n}}(\mathrm{V})$ and $\beta_{\mathrm{n}}(\mathrm{V})$ that were calculated from the experimental data to Equations 9, 10 (from NEURON's ChannelBuilder), using the MATLAB function $f i t()$ :

$$
\begin{gathered}
\alpha_{n}(V)=A\left(1+e^{-k(d-V)}\right) \\
\beta_{n}(V)=A\left(1+e^{-k(d-V)}\right) .
\end{gathered}
$$

Constants $\mathrm{A}, \mathrm{k}$ and $\mathrm{d}$ obtained from these fits (Table 1 ) were used to calculate fitted $n(V), G_{K}(V)$ and $\tau_{n}(V)$ using Equations 11-13. These constants were used as input parameters in ChannelBuilder for potas-

\begin{tabular}{|c|c|c|c|c|c|c|c|c|c|c|c|c|}
\hline \multirow{3}{*}{$\begin{array}{l}\text { Type of } \\
\text { channels }\end{array}$} & \multicolumn{6}{|c|}{ Activation } & \multicolumn{6}{|c|}{ Inactivation } \\
\hline & \multicolumn{3}{|c|}{$\alpha$} & \multicolumn{3}{|c|}{$\beta$} & \multicolumn{3}{|c|}{$\alpha$} & \multicolumn{3}{|c|}{$\beta$} \\
\hline & A & k & d & A & k & d & A & k & d & A & k & d \\
\hline$K$ & 2 & -0.054 & 21 & 0.2 & 0.06 & 40 & - & - & - & - & - & - \\
\hline $\mathrm{Na}$ & 1.9 & 0.14 & -21 & 0.3 & -0.05 & -5 & 0.012 & -0.1 & -9 & 0.07 & 0.2 & -40 \\
\hline
\end{tabular}
sium channels:

$$
\begin{gathered}
n(V)=\frac{\alpha_{n}(V)}{\alpha_{n}(V)+\beta_{n}(V)} \\
G A_{K}(V)=(n(V))^{4}
\end{gathered}
$$

Table 1. Transfer rate functions for voltage-gated potassium and sodium channels parameters are tabulated for the transfer rate functions ( $\alpha$ and $\beta$ ) for voltage-gated potassium and sodium channels

$A, k, d$ are parameters derived from fitting experimental data to Hodgkin-Huxley formalism (see Materials and Methods; Eqs. 6-29) and used in the computational model of a zebrafish dopaminergic neuron shown in Figure 8.

$$
\tau_{n}(V)=\frac{1}{\alpha_{n}(V)+\beta_{n}(V)}
$$

The same approach was used to obtain transfer rate functions for activation and inactivation of voltage-gated sodium channels. In Equations 14-19, $\mathrm{GA}_{\mathrm{Na}}(\mathrm{V})$ and $\mathrm{GI}_{\mathrm{Na}}(\mathrm{V})$ are measured normalized sodium conductances representing steady state activation and inactivation of sodium channels (see Results). $\tau_{\mathrm{m}}(\mathrm{V})$ and $\tau_{\mathrm{h}}(\mathrm{V})$ are measured time constants of activation and inactivation. Using these data in Equations 14-19, we calculated measured activation function $\mathrm{m}(\mathrm{V})$ and activation transfer rate functions $\alpha_{\mathrm{m}}(\mathrm{V}), \beta_{\mathrm{m}}(\mathrm{V})$; and inactivation function $\mathrm{h}(\mathrm{V})$ and inactivation transfer rate functions $\alpha_{\mathrm{h}}(\mathrm{V}), \beta_{\mathrm{h}}(\mathrm{V})$. V is membrane potential:

$$
\begin{gathered}
m(V)=\sqrt[3]{G A_{N a}(V)} \\
h(V)=G I_{N a}(V) \\
\alpha_{m}(V)=\frac{m(V)}{\tau_{m}(V)} \\
\beta_{m}(V)=\frac{1-m(V)}{\tau_{m}(V)} \\
\alpha_{h}(V)=\frac{h(V)}{\tau_{h}(V)} \\
\beta_{h}(V)=\frac{1-h(V)}{\tau_{h}(V)} .
\end{gathered}
$$

Next, we fitted transfer rate functions $\alpha_{\mathrm{m}}(\mathrm{V})$ and $\beta_{\mathrm{m}}(\mathrm{V})$ for activation, and $\alpha_{\mathrm{h}}(\mathrm{V})$ and $\beta_{\mathrm{h}}(\mathrm{V})$ for inactivation of sodium channels that were calculated from experimental data to Equations 20-23 (from NEURON's ChannelBuilder), using the MATLAB function fit():

$$
\begin{gathered}
\alpha_{m}(V)=A\left(\frac{k(V-d)}{\left(1-e^{-k(V-d)}\right)}\right) \\
\beta_{m}(V)=A \mathrm{e}^{k(V-d)} \\
\alpha_{h}(V)=A e^{k(V-d)} \\
\beta_{h}(V)=A\left(\frac{k(V-d)}{\left(1-e^{-k(V-d)}\right)}\right) .
\end{gathered}
$$

Constants A, $\mathrm{k}$ and $\mathrm{d}$ obtained from these fits (Table 1 ) were used to calculate fitted activation and inactivation functions $\mathrm{m}(\mathrm{V})$ and $\mathrm{h}(\mathrm{V})$, 
peak conductances $\mathrm{GA}_{\mathrm{Na}}(\mathrm{V})$ and $\mathrm{GI}_{\mathrm{Na}}(\mathrm{V})$ and time constants $\tau_{\mathrm{m}}(\mathrm{V})$ and $\tau_{\mathrm{h}}(\mathrm{V})$, using Equations 24-29. These constants were used as input parameters in ChannelBuilder for sodium channels:

$$
\begin{gathered}
m(V)=\frac{\alpha_{m}(V)}{\alpha_{m}(V)+\beta_{m}(V)} \\
G A_{N a}(V)=(m(V))^{3} \\
h(V)=\frac{\alpha_{h}(V)}{\alpha_{h}(V)+\beta_{h}(V)} \\
G I_{N a}(V)=h(V) \\
\tau_{m}(V)=\frac{1}{\alpha_{m}(V)+\beta_{m}(V)} \\
\tau_{h}(V)=\frac{1}{\alpha_{h}(V)+\beta_{h}(V)} .
\end{gathered}
$$

\section{Computer model}

A model zebrafish dopaminergic neuron was simulated in the NEURON environment (version 7.7.2; https://www.neuron.yale.edu/neuron/; Hines and Carnevale, 1997), using the graphical user interfaces CellBuilder and ChannelBuilder, without additional code. The model contains a single compartment with a cylindrical soma of $35 \mu \mathrm{m}$ in length and $14.5 \mu \mathrm{m}$ in diameter. Values of specific membrane capacitance and leak conductance density were chosen so that the model neuron showed membrane capacitance and leak conductance values similar to values measured in recorded cells. The reversal potential for the leak current was determined from the resting membrane potential of recorded cells in the presence of TTX. Temperature was set to $22^{\circ} \mathrm{C}$. Active potassium and sodium conductances were added to the model using the ChannelBuilder tool. We modified the default Hodgkin-Huxley squid axon potassium and sodium channels of NEURON, by setting the equation type as "voltage dependence of transfer rate functions $(\alpha, \beta)$," and changing the parameters to values calculated from our experimental data (Table 1).

\section{Results}

Electrophysiological recordings from dopaminergic neurons in zebrafish brain slices reveal spontaneous rhythmic activity We first developed an acute brain slice preparation enabling whole-cell patch clamp recordings from adult zebrafish dopaminergic neurons. To enable reliable identification of dopaminergic neurons in acute brain slices, we exploited a new transgenic line $\operatorname{Tg}$ (th:gal4- $v p 16) ; \operatorname{Tg}($ UAS:egfp), in which GFP is expressed under the transcriptional control of regulatory elements from the gene encoding zebrafish $\mathrm{TH}$, the rate-limiting enzyme in dopamine biosynthesis. Construction and characterization of this line, which harbors a large BAC transgene containing the th gene and upstream sequences, will be described elsewhere. For the present application, we verified that the dopaminergic projection neurons of the adult zebrafish periventricular TPp (diencephalic groups DC2 and DC4) expressed the GFP transgene (Fig. 1A). Confocal microscopy of fixed, axial (transverse) sections through the diencephalon showed extensive co-localization of GFP with $\mathrm{TH}$ in the region of TPp, including the large pear-shaped neurons of interest. For electrophysiological recordings, $200-\mu \mathrm{m}-$ thick axial slices were prepared from freshly-dissected brains embedded acutely in low melting point agarose (Fig. 1B). Two to three sections per brain, which were distinguishable by neuroanatomical landmarks including the diencephalic ventricle and posterior commissure, contained the neuronal populations of interest (Fig. 1B). Dopaminergic DC2/4 neurons were readily identified in these sections by their large pear-shaped soma and anatomic location within TPp adjacent to the diencephalic ventricle (Rink and Wullimann, 2001), and by their strong GFP expression (Fig. 1C). Two to four such cells were located within $50 \mu \mathrm{m}$ of each slice surface, allowing access for microelectrode recordings. Biocytin labeling of a subset of recorded neurons illustrated their characteristic morphology (Fig. 1D). DC2 neurons were located rostrally and their processes projected laterally, whereas DC4 neurons were located caudally and their processes projected dorsolaterally. DC2 and DC4 neurons showed identical pacemaker mechanisms and were analyzed together in this study.

Stable whole-cell patch clamp recordings could be maintained for several hours in this acute slice preparation. All zebrafish DC2/4 neurons recorded showed robust spontaneous pacemaker activity (Fig. $1 E$ ). Figure $1 E, F$ show an example in which a cell discharged APs rhythmically at a mean rate of $2.12 \pm 0.41 \mathrm{~Hz}$ for almost $3 \mathrm{~h}$ of recording. The frequency of autonomous activity varied between 1.2 and $3.2 \mathrm{~Hz}$ over the duration of this prolonged recording. However, over short periods of time, there was very little variation in the instantaneous discharge frequency (as reflected in the coefficient of variation of the interspike interval; Fig. $1 F$ ) and we found no evidence of bursting. The mean membrane potential during interspike intervals remained stable throughout the recording (Fig. $1 F$ ), although there was a modest decrease in AP amplitude during prolonged experiments (Fig. $1 F$ ).

Together, these data show that acute brain slices from $\operatorname{Tg}(t h$ : gal4-vp16); $\operatorname{Tg}(U A S: e g f p)$ zebrafish can be used to obtain stable patch clamp recordings from adult zebrafish DC2/4 dopaminergic neurons. This new experimental preparation enabled us to investigate the electrophysiological properties of dopaminergic neurons and to interrogate the mechanisms underlying their cell-intrinsic pacemaker activity.

\section{Electrophysiological properties of zebrafish dopaminergic neurons}

We next determined the electrophysiological properties of a sample of 130 zebrafish DC2/4 dopaminergic neurons (Fig. 2). The mean $\pm \mathrm{SD}$ membrane potential during interspike intervals was $-51.2 \pm 2.3 \mathrm{mV}$ (Fig. $2 A, B$ ). The mean threshold potential was $-35.9 \pm 3.9 \mathrm{mV}$ (Fig. 2A,B). In individual neurons, the threshold potential was only $15.4 \pm 2.6 \mathrm{mV}$ depolarized relative to the mean membrane potential during interspike intervals. APs peaked at $29.5 \pm 9.1 \mathrm{mV}$, followed by a pronounced fast AHP to $-62.9 \pm 3.1 \mathrm{mV}$ and a slow AHP (Fig. $2 A, B$ ). AP amplitude, measured from peak to most negative AHP, was $92.3 \pm 11.1 \mathrm{mV}$. AP width measured at half-amplitude was $1.3 \pm 0.23 \mathrm{~ms}$. There was a negative correlation between AP amplitude and width: APs with smaller amplitudes were broader $(r=-0.65$, $p<0.001$ ). Broad APs with a marked AHP and depolarized AP threshold are typical of dopaminergic neurons from multiple different species (Grace and Onn, 1989; Yung et al., 1991). Mean \pm SD input resistance was $475 \pm 174 \mathrm{M} \Omega$ and membrane capacitance $80 \pm 25 \mathrm{pF}$; input resistance and capacitance correlated as expected with neuron size, such that larger neurons showed higher capacitance and lower resistance (Fig. 2C,D).

All recorded neurons generated rhythmic autonomous APs with mean frequency $3.1 \pm 1.5 \mathrm{~Hz}$ and low variability (mean coefficient of variation of interspike intervals $0.13 \pm 0.12$ ). There was a negative correlation between firing frequency and 
A
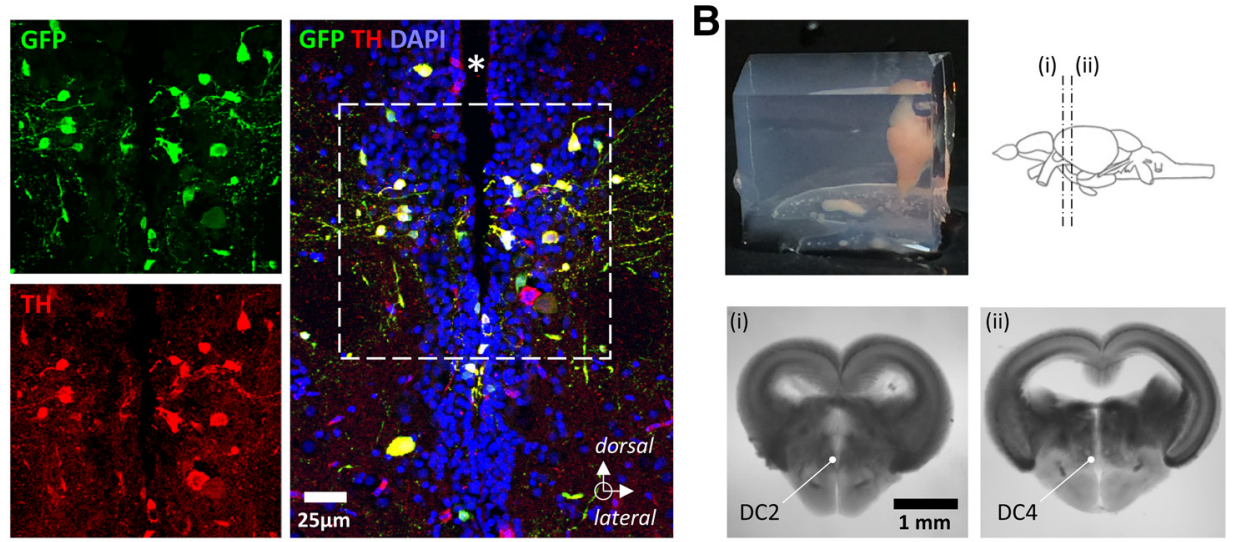

C

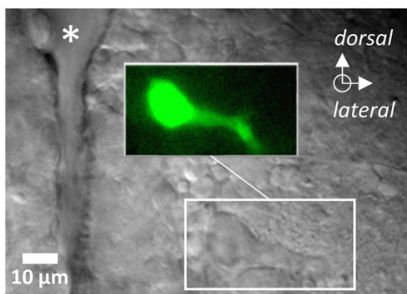

D
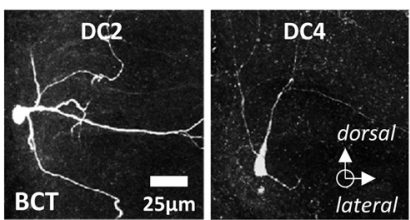

E
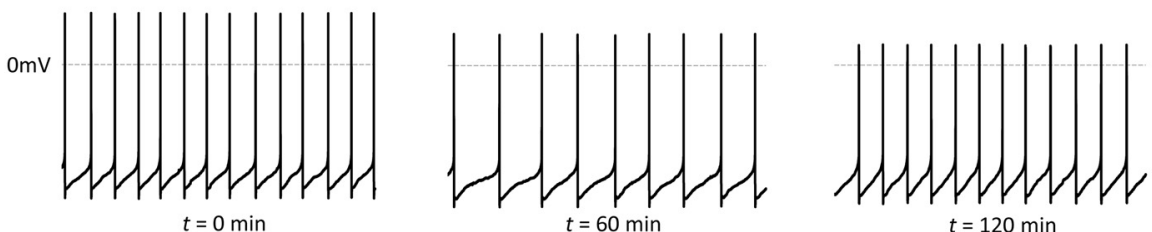

$t=120 \mathrm{~min}$
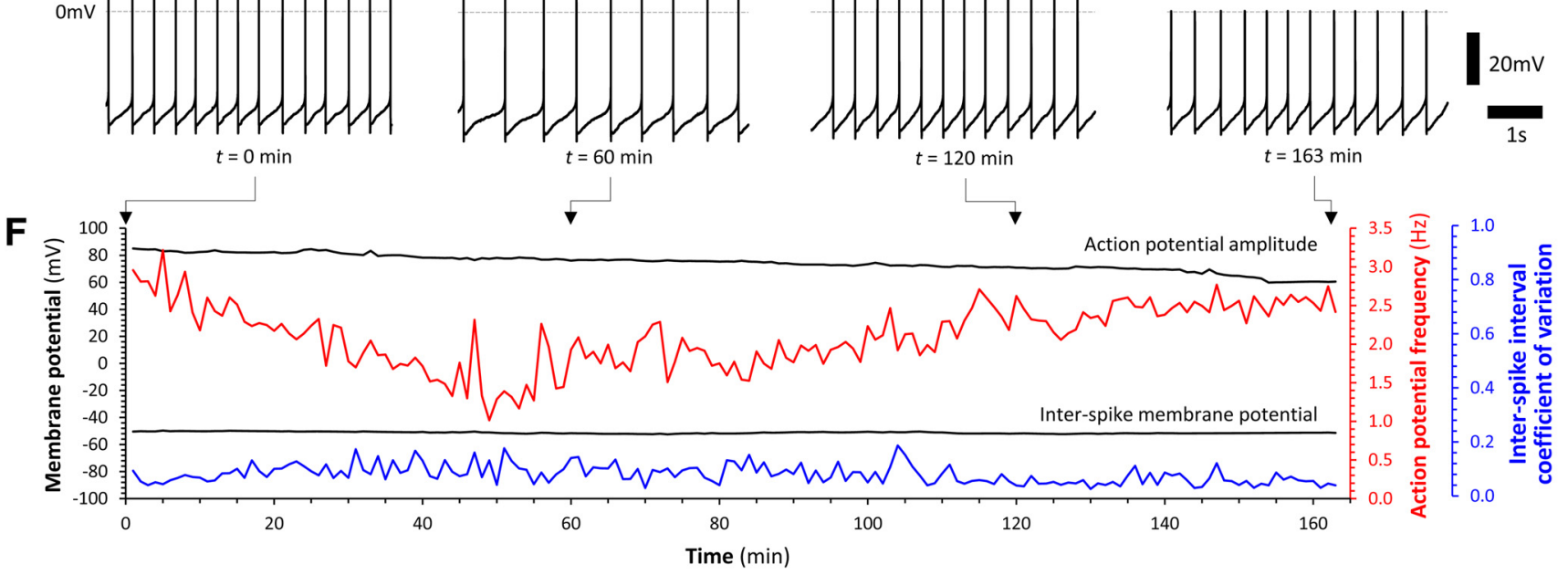

Figure 1. Acute brain slice preparation from a novel transgenic line reveals robust pacemaker activity in zebrafish DC2/4 dopaminergic neurons. A, Confocal z-plane projection of an axial section from an adult Tg(th:ga/4); $\operatorname{Tg}($ UAS:egfp) zebrafish brain, labeled by immunofluorescence for TH (red) and GFP (green), with a nuclear counterlabel (DAPl; blue). The images show the TPp adjacent to the diencephalic ventricle $(*)$. To the left, individual channels are shown separately for the boxed region in the main panel, illustrating the extensive colocalization between the GFP marker and endogenous TH. B, Zebrafish brains were embedded in low melting temperature agarose and 200- $\mu \mathrm{m}$ axial (transverse) sections were made. The planes (upper right) and appearances (lower) of the slices containing dopaminergic neurons are shown. The approximate locations of DC2 and DC4 neurons are indicated. C, Infrared DIC micrograph showing a dopaminergic neuron (white box) in an acute brain slice. The neuron is readily identified by its morphology and position adjacent to the diencephalic ventricle (*) and by its strong expression of the GFP transgene, visible by epifluorescence microscopy (inset). D, A subset of neurons was filled with biocytin during recording and imaged by confocal microscopy following incubation with Alexa Fluor 555-streptavidin. Example confocal z-plane projections illustrate the characteristic morphology of DC2 (left) and DC4 (right) dopaminergic neurons. $\boldsymbol{E}$, Membrane potential traces showing sustained pacemaker activity in a dopaminergic neuron at times between 0 and 163 min after establishing a whole-cell patch clamp recording. $\boldsymbol{F}$, The AP amplitude and interspike membrane potential (black traces, left axis), AP frequency (red) and coefficient of variation of the interspike intervals (blue; right axes) are shown over time for the cell from panel $\boldsymbol{E}$.

coefficient of variation, so that neurons with higher pacemaker frequency also showed enhanced regularity (Fig. $2 F$ ). However, the frequency of spontaneous activity did not correlate with neuronal size (Fig. $2 E$ ), input resistance, membrane potential during interspike intervals (Fig. 2G), or AHP (Fig. $2 H$ ).

Together, these data show that zebrafish DC2/4 dopaminergic neurons express robust pacemaker activity at $2-4 \mathrm{~Hz}$ with high rhythmicity and low variability. Their basic electrophysiological properties are consistent with values reported for dopaminergic neurons in other species (see Discussion).

\section{Properties and modulation of the zebrafish dopaminergic pacemaker}

To elucidate the mechanisms underlying autonomous activity, we next analyzed the voltage dependence of the pacemaker during injection of long ( $0.5 \mathrm{~s}$ ) depolarizing current steps (Fig. $3 A$ ). The frequency of firing increased monotonically with current injection. The relationship was close to linear with current steps up to 300$400 \mathrm{pA}$, and the instantaneous frequency (reciprocal of the first interspike interval) reached $\approx 80 \mathrm{~Hz}$ at $400 \mathrm{pA}$ (Fig. $3 B$, blue line and symbols). Over this range, the frequency and amplitude of the evoked APs decreased slightly during the first 30-60 ms after the onset of each step but then remained stable without significant adaptation, so that mean discharge frequency (number of APs during the time period in which they could be detected) also increased almost linearly to $\approx 60 \mathrm{~Hz}$ at $400 \mathrm{pA}$ (Fig. $3 B$, black line and symbols). With further increases in the amplitude of the current step above $400 \mathrm{pA}$, discharge frequency and AP amplitude showed progressively stronger adaptation. The maximal instantaneous initial frequency in response to larger current steps was $\approx 130 \mathrm{~Hz}$ and maximal mean frequency was $\approx 85 \mathrm{~Hz}$ (Fig. $3 A, B$ ). However, depolarization block, a characteristic sign of voltage-gated sodium channel inactivation, was provoked by the largest depolarizing currents, so that $\mathrm{AP}$ discharges were not maintained for the duration of the step (Fig. 3A).

Hyperpolarizing current steps suppressed pacemaker activity completely (Fig. $3 A, C$ ) and caused sustained membrane hyperpolarization without sag. The voltage-current relationship for 
A
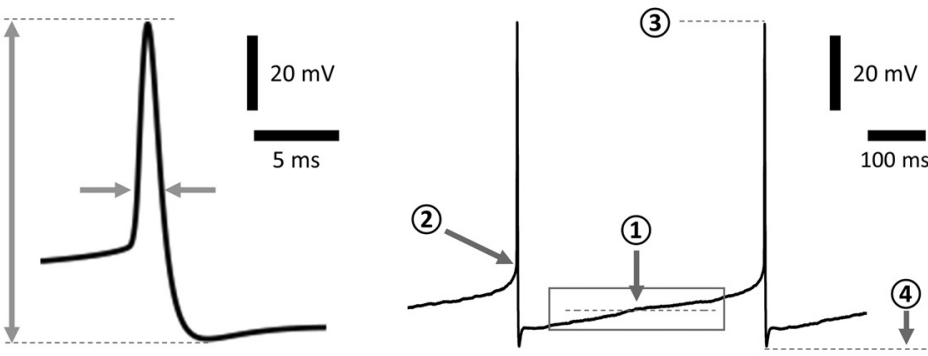

C

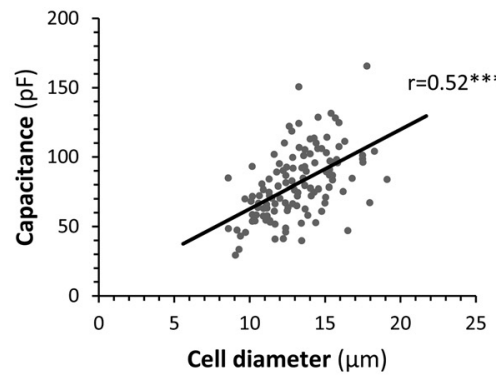

$\mathbf{F}$

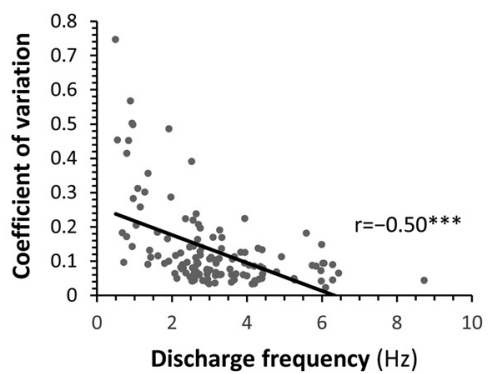

D

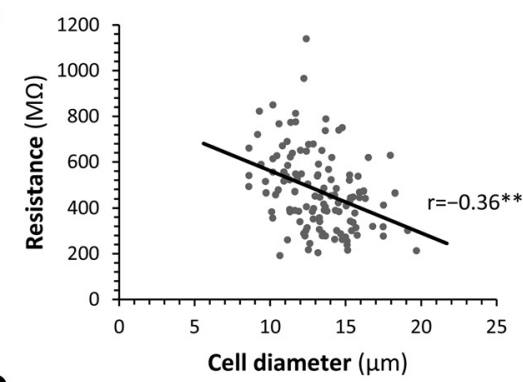

G

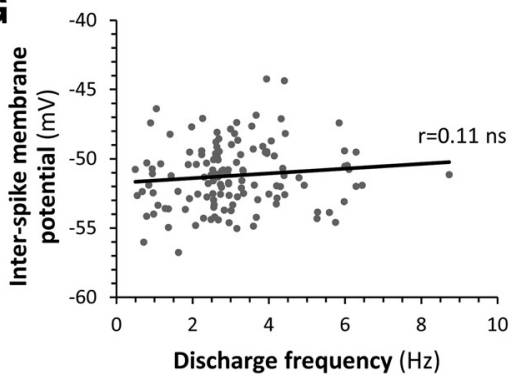

B

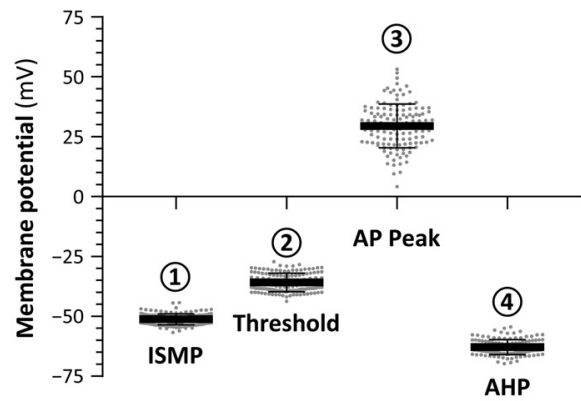

E

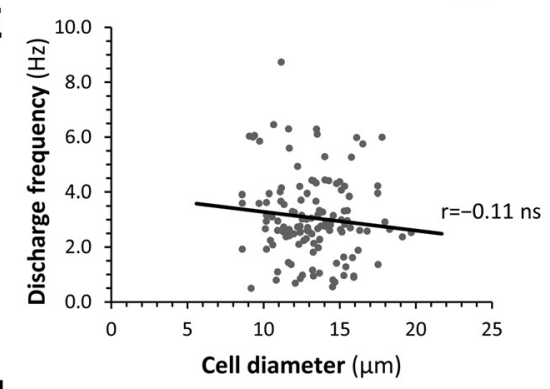

H

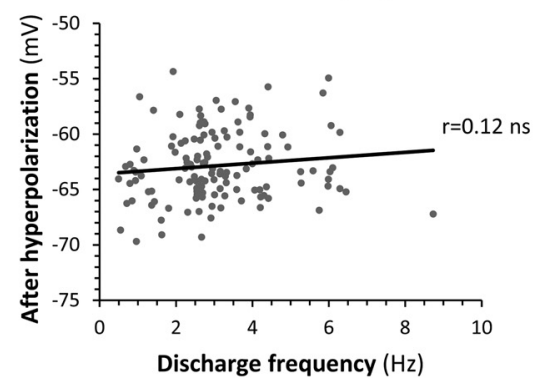

Figure 2. Electrophysiological properties of zebrafish DC2/4 dopaminergic neurons. $A$, An averaged AP from a dopaminergic neuron is shown on the left; arrows indicate how amplitude and width at half amplitude were measured. Two consecutive APs are shown on the right. Arrows indicate how interspike membrane potential (ISMP; (1)), AP threshold (2)), AP peak (3) and AHP (4) were measured. $\boldsymbol{B}$, Scatter plots showing values for each of these parameters from a sample of $n=130 \mathrm{DC} / 4$ neurons. Data points show values from individual neurons, lines and bars show mean $\pm S D$. $\boldsymbol{C}-\boldsymbol{H}, x / y$ scatter plots showing the relationship between: $(\boldsymbol{C})$ cell diameter and membrane capacitance, $(\boldsymbol{D})$ cell diameter and input resistance, $(\boldsymbol{E})$ cell diameter and AP frequency, $(\boldsymbol{F})$ AP frequency and coefficient of variation of the interspike interval, $(\boldsymbol{G})$ AP frequency and membrane potential during interspike intervals, and $(\boldsymbol{H})$ AP frequency and AHP. Data points show values from individual neurons $(n=130)$, lines were derived by linear regression $\left({ }^{*} p<0.05,{ }^{* *} p<0.01\right.$, ${ }^{* * *} p<0.001$, ns, not significant).

hyperpolarizing steps was near to linear, allowing extrapolation of a resting membrane potential value of $-51.8 \mathrm{mV}$ (Fig. $3 \mathrm{C}$, green line). This corresponds closely to the mean value of $-51.2 \pm 2.3 \mathrm{mV}$ measured during interspike intervals.

The absence of membrane potential sag during injection of a prolonged hyperpolarizing current suggested that zebrafish dopaminergic neurons lack a hyperpolarization-activated current $\left(\mathrm{I}_{\mathrm{h}}\right)$ mediated by HCN channels. We confirmed that HCN channels do not contribute to the autonomous activity or hyperpolarization responses of zebrafish dopaminergic neurons by using an $\mathrm{HCN}$ antagonist, ZD7288 (Fig. 3D-F, $n=12$ neurons). Application of ZD7288 at $50 \mu \mathrm{M}$ did not change either pacemaker activity (Fig. $3 D, E$ ), or the response to a hyperpolarizing current step (Fig. $3 F$ ).

Together, these data suggest that hyperpolarization-activated currents do not contribute to the pacemaker in zebrafish dopaminergic neurons. Discharge frequency can be increased by depolarizing currents, and decreased or suspended by hyperpolarizing currents, suggesting that synaptic inputs to these cells in the intact CNS can modulate their autonomous activity.

\section{Voltage-gated sodium channels are essential for pacemaker activity}

Our observations, including: (1) near-linear changes in discharge frequency with moderate (up to 300-400 pA) depolarizing currents; (2) near-linear changes in membrane potential with hyperpolarizing current steps; and (3) absence of $\mathrm{I}_{\mathrm{h}}$, together imply that voltage-gated sodium channels mediate the only inward current that is critical to the generation of pacemaker activity in zebrafish dopaminergic neurons. If this is true, blockade of voltage-gated sodium channels should eliminate pacemaker activity, including subthreshold oscillations of the membrane potential. Alternatively, if additional conductances participate in the pacemaker mechanism, blockade of sodium channels should reveal subthreshold membrane potential oscillations or calcium spikes, which have been observed in some types of mammalian dopaminergic neuron (Ping and Shepard, 1996; Chan et al., 2007; Puopolo et al., 2007).

To distinguish these possibilities, we added the voltage-gated sodium channel blocker TTX ( $1 \mu \mathrm{M}$ final concentration) to the extracellular medium during recording ( $n=16$ experiments). In the example shown in Figure $4 A$, the frequency of autonomous spiking gradually decreased, and firing became less regular during TTX wash-in. Episodes of irregular firing were interrupted by progressively longer silent periods (Fig. 4A). As expected, the AP amplitude decreased gradually during TTX wash-in (Fig. 4B). In addition, the rate of membrane depolarization during the interspike interval also gradually decreased (Fig. $4 B, C$ ), suggesting that sodium channels contribute to the spontaneous membrane depolarization that drives pacemaker activity. Interestingly, as the proportion of sodium channels blocked by TTX increased during 


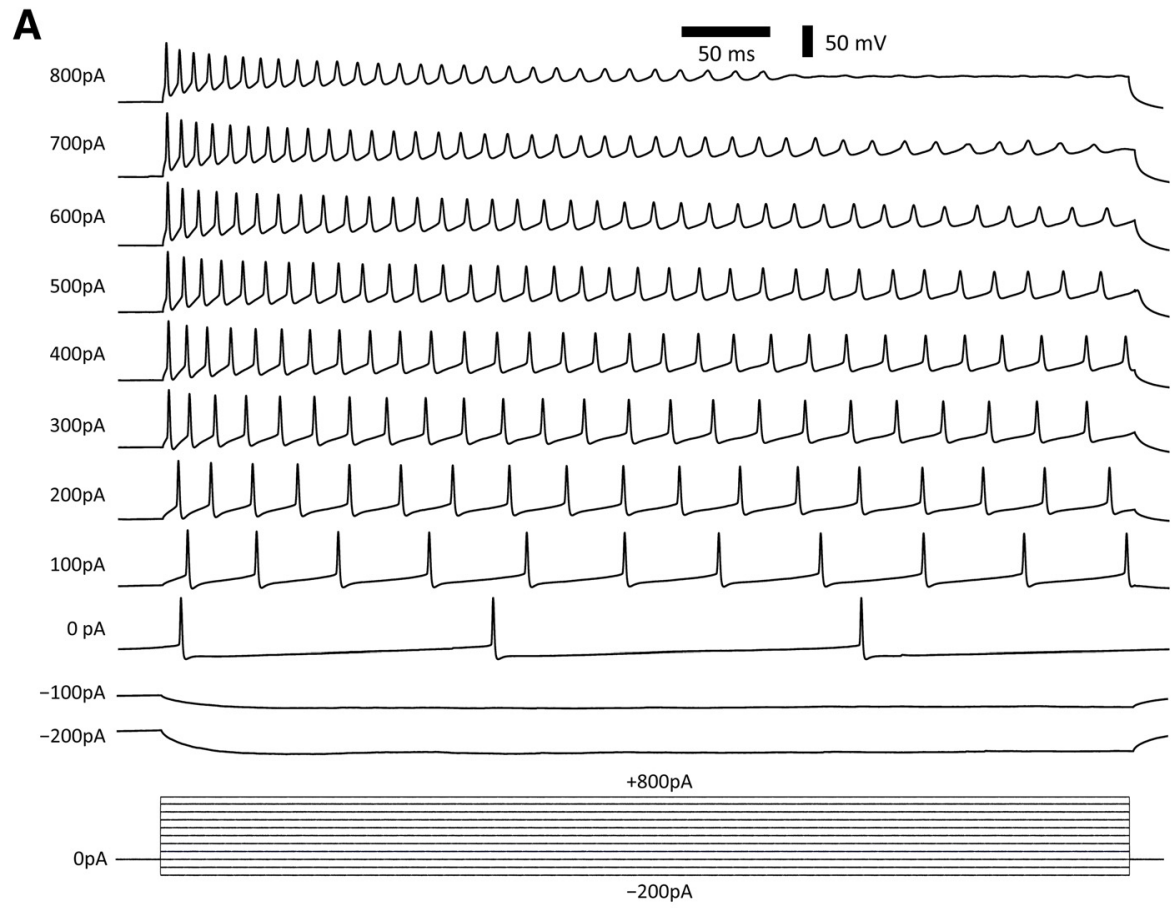

D

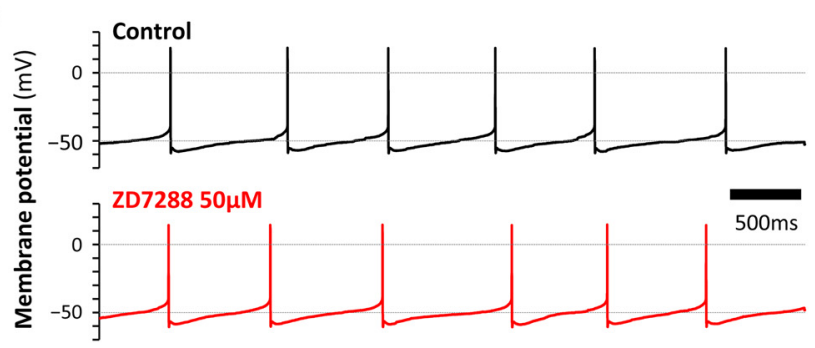

B
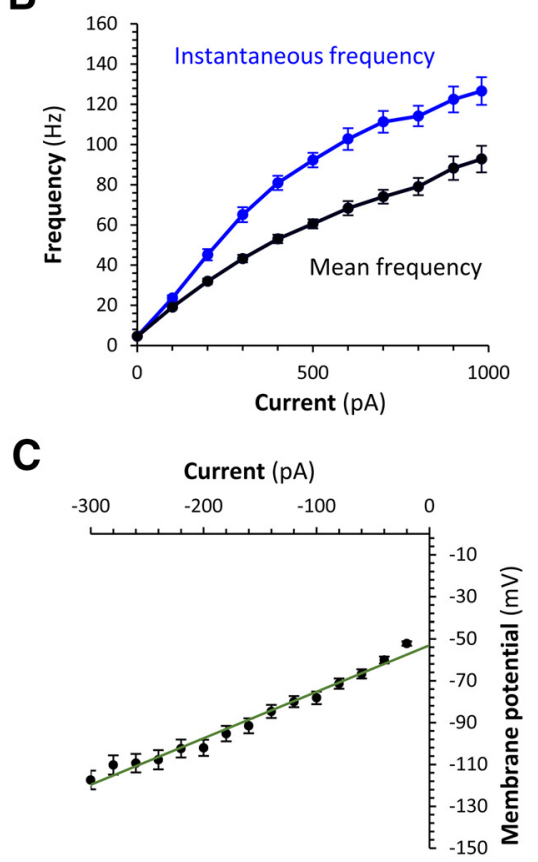

F

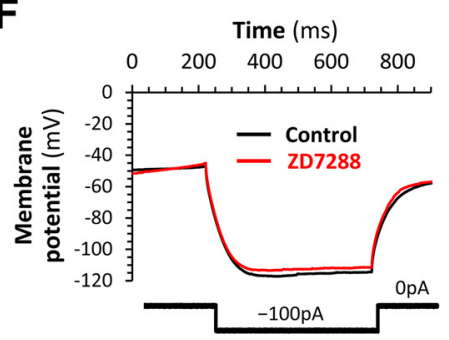

Figure 3. The pacemaker in zebrafish DC2/4 dopaminergic neurons is voltage-dependent but does not involve HCN channels. $\boldsymbol{A}$, Membrane potential/time traces showing the responses of a dopaminergic neuron to hyperpolarizing and depolarizing current steps between -200 and $+800 \mathrm{pA}$, as indicated below. $\boldsymbol{B}$, Relationship between the amplitude of depolarizing current steps and instantaneous frequency (reciprocal of the first interspike interval; blue) or mean frequency (black); markers show mean \pm SE for $n=16$ neurons. $\boldsymbol{C}$, Relationship between the amplitude of injected hyperpolarizing current and membrane potential (markers show mean \pm SE for $n=16$ neurons). The best fit line (green) was derived by linear regression; its extrapolation to the $y$-axis was used to estimate resting membrane potential. $\boldsymbol{D}, \boldsymbol{E}$, Effect of ZD7288, an antagonist of HCN channels, on pacemaker activity in DC2/4 dopaminergic neurons. $\boldsymbol{D}$, Example membrane potential traces of a neuron under control conditions (above; black) and after bath application of ZD7288 at a final concentration of $50 \mu \mathrm{m}$ (below; red). $\boldsymbol{E}$, Spontaneous discharge frequency of neurons under control conditions (black) and following application of $50 \mu \mathrm{m}$ ZD7288 (red). Data points show individual neurons, bars shown mean \pm SE ( $n=12$ neurons). ns, not significant. $\boldsymbol{F}$, The membrane potential traces show the responses of the same cell shown in $\boldsymbol{D}$ to injection of a hyperpolarizing current step ( $-100 \mathrm{pA}$, timing is shown below the membrane potential trace) both under control conditions (black) and after application of ZD7288 (red).

TTX wash-in, the firing frequency evoked by 80 -pA depolarizing current steps did not change $(18.71 \pm 6.1 \mathrm{~Hz}$ baseline vs $17.9 \pm$ $6.1 \mathrm{~Hz}$ during TTX wash-in; $p=0.17$ ). Compromised sodium channel function was thus sufficient to suspend pacemaker activity, without altering the discharge of APs in response to depolarizing steps. Together, these data indicate that voltage-gated sodium channels, in addition to mediating the AP upstroke, are also critical for producing spontaneous depolarization during the interspike interval underlying pacemaker activity. Moreover, after complete blockade of voltage-gated sodium channels, the membrane potential became stable; no oscillations or calcium spikes were observed, either at the resting membrane potential or during injection of depolarizing current (Fig. 4A,D). This suggests that TTX-sensitive sodium channels mediate the only voltage-gated inward (depolarizing) current contributing to the pacemaker activity of zebrafish dopaminergic neurons.

Compared with the interspike membrane potential during spontaneous pacemaker activity, the resting membrane potential in dopaminergic neurons treated with TTX was relatively depolarized, both at baseline and during injection of depolarizing current steps (Fig. 4D, overlay of baseline and post-TTX traces). In contrast, the membrane potential of the same cells during hyperpolarizing pulses (which suppressed spiking) did not differ significantly between baseline and post-TTX conditions (Fig. $4 D-F$ ). This suggests that hyperpolarizing conductances (likely mediated by potassium channels) are activated by APs during pacemaker activity, and strongly influence the mean membrane potential during interspike intervals. Blockade of APs with TTX consequently prevents secondary activation of potassium channels, leading to apparent depolarization of the resting membrane potential (Figs. $4 D, 5 A, B$ ).

To further clarify ionic contributions to electrogenesis in zebrafish dopaminergic neurons, we combined application of specific channel blockers with the use of modified extracellular solutions that were nominally free of either sodium or calcium ions. Baseline measurements of pacemaker activity, interspike interval membrane potential $(-49.9 \pm 2.4 \mathrm{mV})$ and threshold potential $(-36 \pm 4.6 \mathrm{mV} ; n=23)$ were identical in these experiments to the recordings shown in Figures $2 B, 3 C, 5 A, B$. As 
A Control

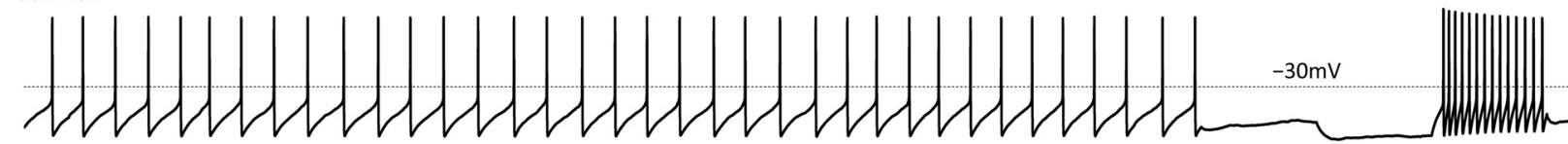

TTX wash in
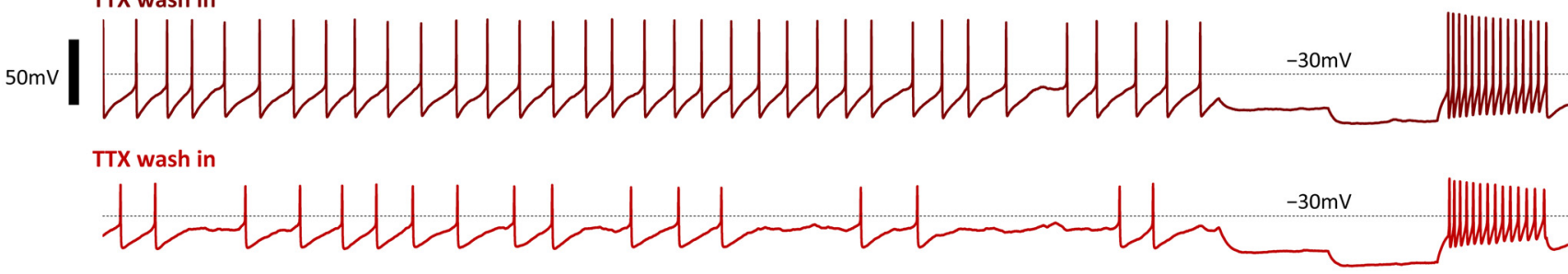

$\begin{array}{lll}\text { TTX } & -30 \mathrm{mV}\end{array}$

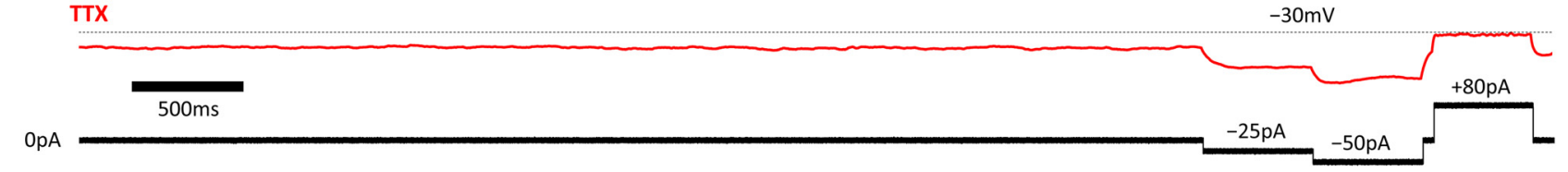

B
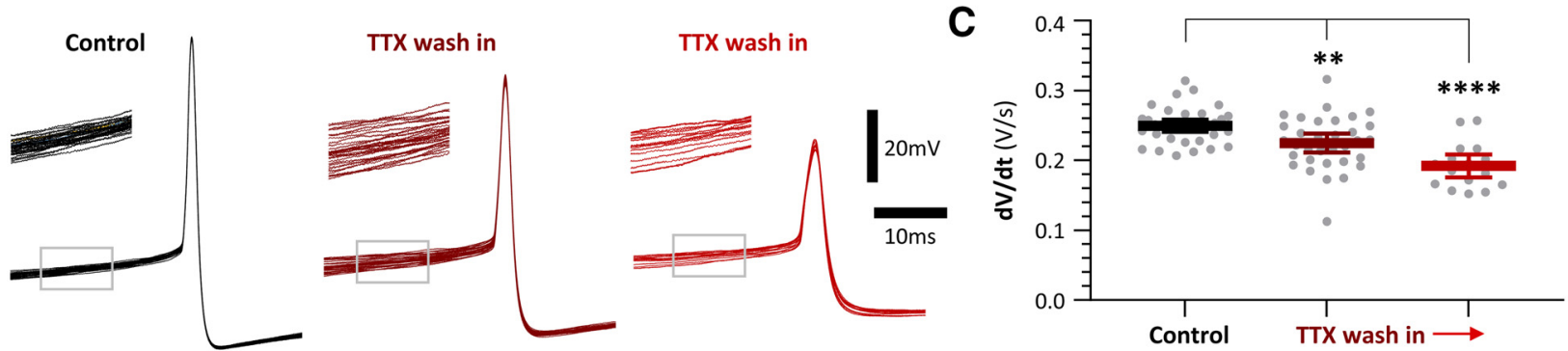

D

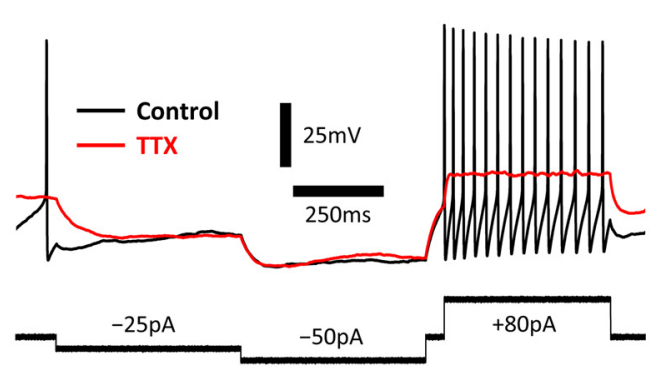

$\mathbf{E}$

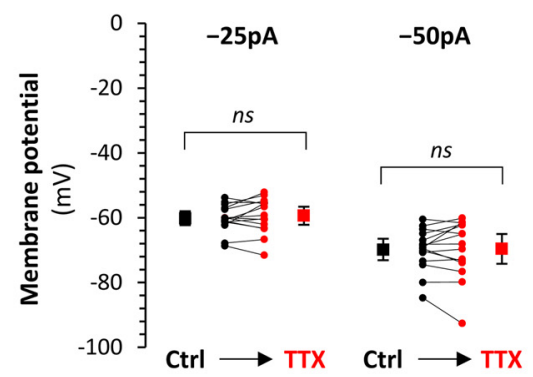

$\mathbf{F}$

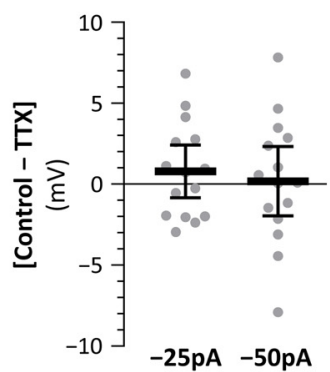

Figure 4. Progressive loss of autonomous pacemaker activity in zebrafish DC2/4 dopaminergic neurons during blockade of voltage-gated sodium channels. $\boldsymbol{A}$, Membrane potential traces from a dopaminergic neuron, showing spontaneous pacemaker activity without current injection, and responses to injection of hyperpolarizing and depolarizing current steps $(-25,-50$, and $+80 \mathrm{pA}$ ), under control conditions (black) and during TTX wash-in (increasing red). The lower trace shows the timing and amplitude of the injected current. $\boldsymbol{B}$, Traces of spontaneous APs from panel $\boldsymbol{A}$, during control conditions and TTX wash-in, are superimposed. Insets show the boxed regions of the traces at a larger scale, to illustrate the effects of TTX on the slope and variability of the interspike membrane potential. C, The rate of membrane potential depolarization in the 20-ms window before onset of the AP [defined by (peak $-23 \mathrm{~ms}$ ) to (peak $-3 \mathrm{~ms}$ )], under control conditions and during TTX wash-in. Bars show mean \pm SE of $n=17-35$ interspike intervals; $* * p<0.01, * * * * p<0.0001$ compared with control, one-way ANOVA with Dunnett's post hoc test. $\boldsymbol{D}$, Responses of the neuron shown in panel $\boldsymbol{A}$ to current steps before (black) and after (red) TTX are superimposed for comparison. The responses to hyperpolarizing steps are unchanged by TTX; the membrane depolarization in response to a depolarizing step is increased following TTX. $\boldsymbol{E}$, The membrane potential during hyperpolarizing current steps of $-25 \mathrm{pA}$ (left) or $-50 \mathrm{pA}$ (right) is shown for $n=15$ different dopaminergic neurons, under control conditions (black) and after application of TTX (red). Small circular markers show values for individual neurons, with control and post-TTX values connected by lines; large square markers show mean \pm SE for all 15 neurons (ns, not significant; control vs TTX, two-tailed paired $t$ test). $F$, For each neuron from panel $\boldsymbol{C}$, the change in membrane potential during hyperpolarizing steps ( $-25 \mathrm{pA}$, left or $-50 \mathrm{pA}$, right) after TTX application was calculated. Gray data points show 15 individual dopaminergic neurons, bars show mean difference \pm SE for $n=15$ neurons.

shown in Figure 4, following blockade of voltage-gated sodium channels with $1 \mu \mathrm{M}$ TTX, the resting membrane potential was depolarized (mean $-44.04 \pm 4.4 \mathrm{mV} ; p<0.001$ ) compared with the interspike membrane potential before TTX application. Exchanging the extracellular solution for sodium-free (sodium substituted with choline $+1 \mu \mathrm{M}$ TTX; $-45.5 \pm 4.5 \mathrm{mV}, n=7$ ), or calcium-free ( $+1 \mu \mathrm{M}$ TTX; $-41.4 \pm 4.4 \mathrm{mV}, n=3)$ media did not alter the resting membrane potential further (Fig. $5 B$ ). However, in the presence of $1 \mu \mathrm{M}$ TTX, addition of $20 \mathrm{~mm}$ TEA and recording with a cesium-based intracellular solution to block potassium channels resulted in further membrane depolarization (Fig. 5B). This is consistent with a potassium leak current contributing significantly to the resting membrane potential. However, the depolarized membrane potential in the presence of TTX alone is consistent with a relatively low density of potassium leak channels in zebrafish dopamine neurons, as has also been reported in the mammalian SNc and ventral tegmental area (VTA; Talley et al., 2001). This might explain the high input resistance of zebrafish DC2/4 dopaminergic neurons (mean $\pm \mathrm{SE}$ was $475 \pm 15 \mathrm{M} \Omega$; compared with $394 \pm 21$ $\mathrm{M} \Omega$ in the mammalian SNc; Häusser et al., 1995). 
A

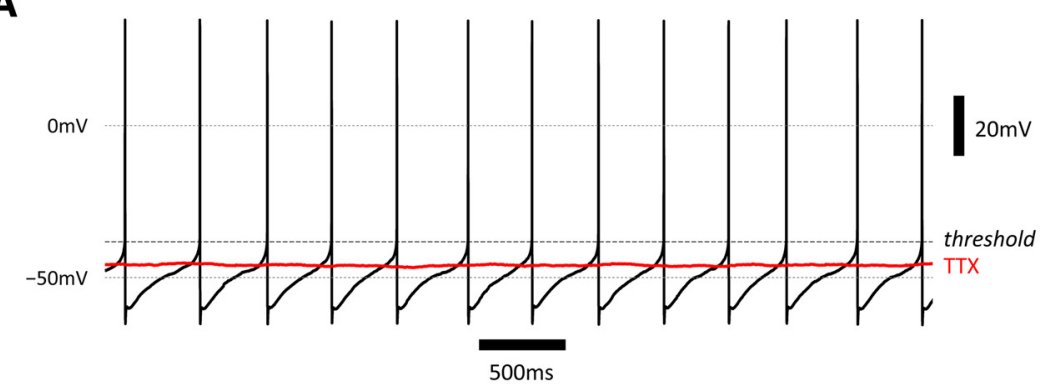

B

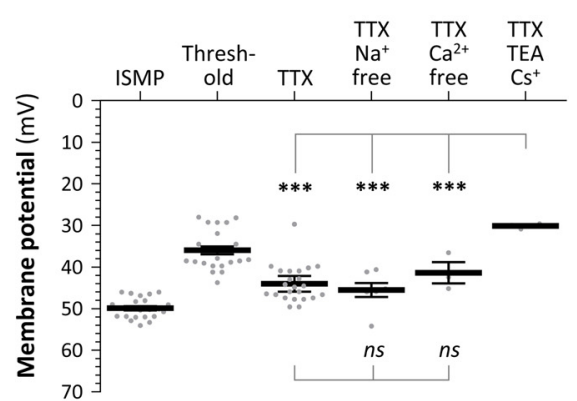

C
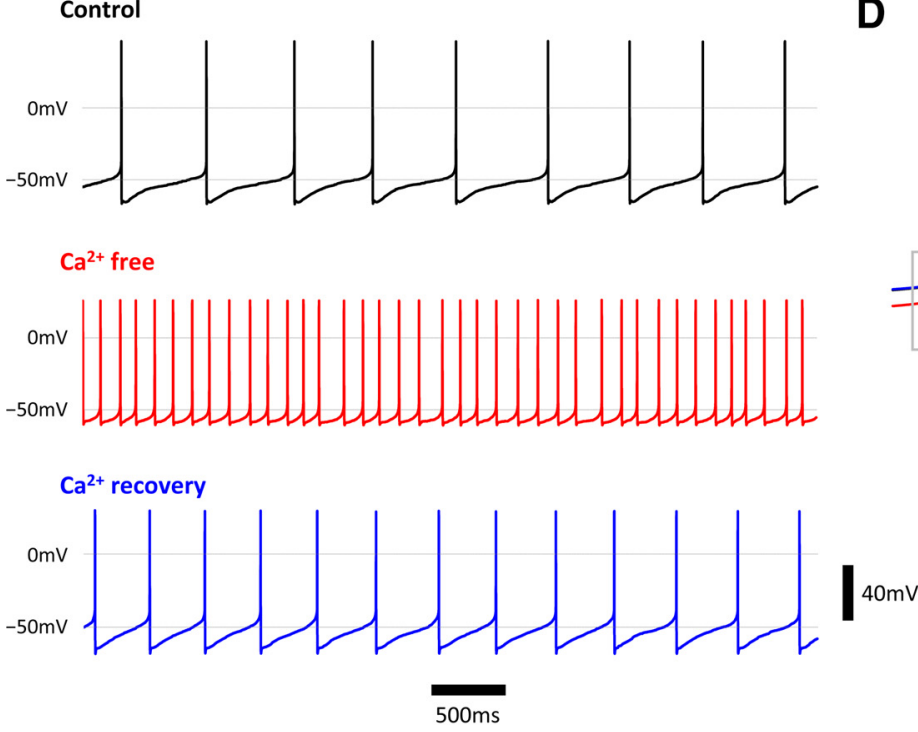

D

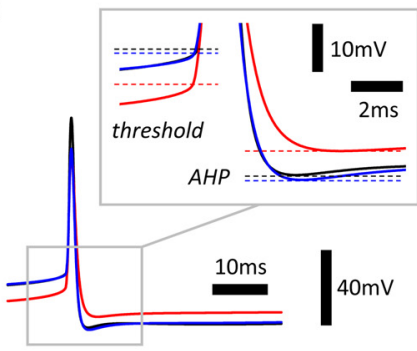

F

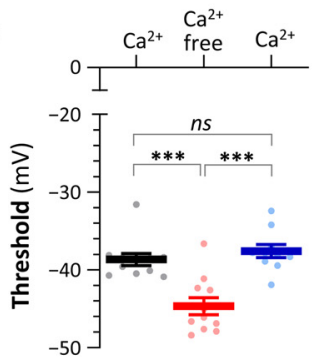

E

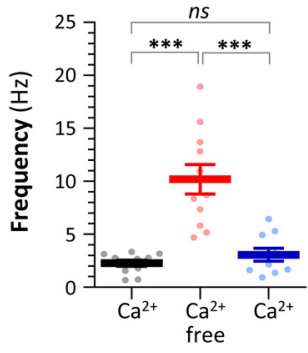

G

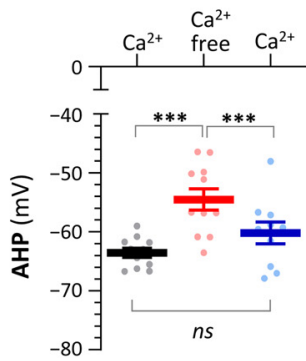

Figure 5. Role of $\mathrm{Na}^{+}, \mathrm{K}^{+}$, and $\mathrm{Ca}^{2+}$ ions in the resting membrane potential and autonomous pacemaker activity of zebrafish DC2/4 dopaminergic neurons. $\boldsymbol{A}$, Membrane potential trace from a dopaminergic neuron under control conditions (black; AP threshold is indicated by a dashed line) and after application of TTX (red). $\boldsymbol{B}$, Scatterplot showing membrane potential during interspike intervals (ISMP) and AP threshold under control conditions $(n=23)$, membrane potential after TTX application ( $n=23)$, and membrane potential after TTX application followed by perfusion with $\mathrm{Na}^{+}$-free $(n=7)$ or $\mathrm{Ca}^{2+}$-free $(n=3)$ extracellular solution. The final group shows membrane potential in extracellular solution with TTX and TEA, in cells recorded with a cesium-based intracellular solution $(n=4)$. Data points show values for individual neurons, lines show mean \pm SE; $* * * p<0.001$ (one-way ANOVA with Tukey's multiple comparisons test). $C$, Membrane potential trace showing pacemaker activity of a dopaminergic neuron under control conditions (black), in Ca ${ }^{2+}$-free extracellular solution (red), and after restoration of control conditions (blue). $\boldsymbol{D}$, Averaged APs from the traces in panel $\boldsymbol{C}$ are superimposed (the color key is identical). The inset panel shows the boxed area in the main figure at a larger scale, to illustrate how threshold potential and AHP change in the absence of $\mathrm{Ca}^{2+}$ and recover on replacement of $\mathrm{Ca}^{2+}$. $\boldsymbol{E}-\boldsymbol{G}$, Scatterplots showing $(\boldsymbol{E})$ AP frequency, $(\boldsymbol{F})$ threshold potential, and $(\boldsymbol{G})$ AHP, under control conditions (black), $\mathrm{Ca}^{2+}$-free extracellular solution (red) and following return to control conditions (blue). Data points show individual neurons ( $n=11$ ); lines show mean \pm SE; $* * * p<0.001$ (repeated measures one-way ANOVA with Tukey's multiple comparisons test). ns, not significant.

\section{Role of calcium in pacemaker frequency}

Pacemaker frequency increased approximately fourfold after removal of calcium from the extracellular medium (example shown in Fig. $5 C ; n=11$; baseline $2.3 \pm 0.95 \mathrm{~Hz}$ vs calcium-free $10.2 \pm 4.6 \mathrm{~Hz} ; p<0.001)$. This was accompanied by a shift in threshold potential to a more hyperpolarized value that was closer to the interspike membrane potential (baseline $-38.7 \pm$ $2.6 \mathrm{mV}$ vs calcium-free $-44.7 \pm 3.7 \mathrm{mV} ; p<0.001$ ), and a decreased AHP (baseline $-63.5 \pm 2.6 \mathrm{mV}$ vs calcium-free $-54.5 \pm 6.0 \mathrm{mV} ; p<0.001)$. After calcium-free solution was replaced with standard extracellular solution containing calcium, these parameters returned to baseline values.

Since no subthreshold oscillations of membrane potential were observed when sodium channels were blocked by TTX, the mechanisms that mediate the effects of calcium removal are likely activity-dependent, and involve modulation of the TTXsensitive pacemaker. The shift of threshold potential to a more hyperpolarized value could be attributable to a direct action of extracellular calcium ions on sodium channel gating (Armstrong and Cota, 1991), and might be the main factor contributing to the increased firing frequency. In contrast, the decreased AHP in calcium-free solution could be caused by attenuation of a potassium conductance which is sensitive to intracellular calcium. Decrease of the AHP may have a bi-directional effect on firing frequency: it will tend to increase firing frequency because the cell remains closer to the threshold, but it will also tend to decrease firing frequency because of slower recovery of sodium channels from inactivation at less hyperpolarized potentials (Baranauskas, 2007). The effects of extracellular and intracellular calcium might be dissociated by blocking cellular calcium entry through voltage-gated calcium channels. Addition of $50 \mu \mathrm{M}$ $\mathrm{Cd}^{2+}$ (a broad-spectrum calcium channel blocker) to the extracellular medium significantly reduced both the fast (baseline $-62.6 \pm 3.9 \mathrm{mV}$ vs post- $\left.\mathrm{Cd}^{2+}-57.2 \pm 5.5 \mathrm{mV} ; n=8 ; p<0.05\right)$ and slow (baseline $-58.5 \pm 3.0 \mathrm{mV}$ vs post- $\mathrm{Cd}^{2+}-52.5 \pm 4.1 \mathrm{mV}$, $n=8, p<0.001$; Fig. $6 C$ ) components of the AHP, but did not influence the threshold potential (baseline $-35.0 \pm 4.4 \mathrm{mV}$ vs post$\left.\mathrm{Cd}^{2+}-35.4 \pm 4.7 \mathrm{mV} ; n=8\right)$. Cadmium also reduced rather than increased the discharge frequency (baseline $3.47 \mathrm{~Hz}$ vs post-Cd ${ }^{2+}$ $2.12 \mathrm{~Hz}, p<0.04$ ), possibly by slowing the recovery of sodium channels from inactivation because of the smaller AHP. This suggests that intracellular calcium can modulate the AHP, but these 
A

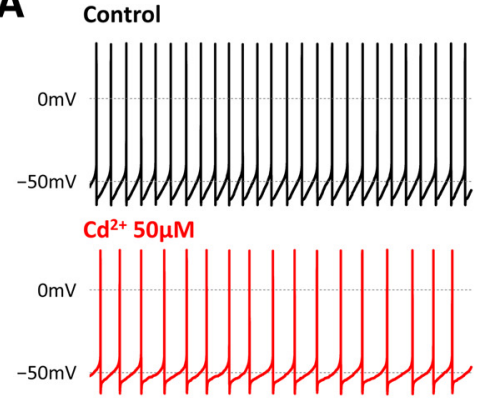

D
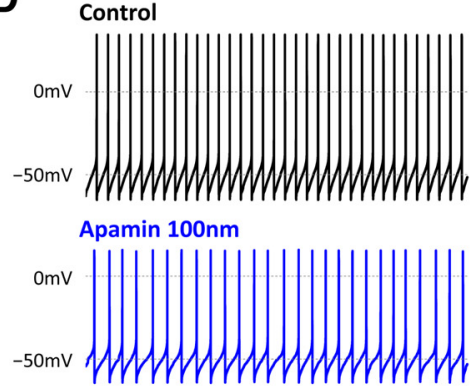

G
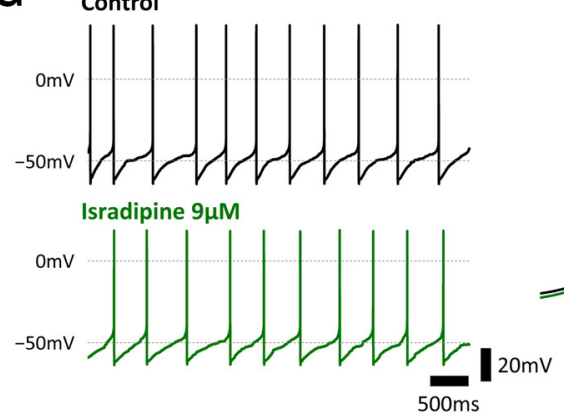
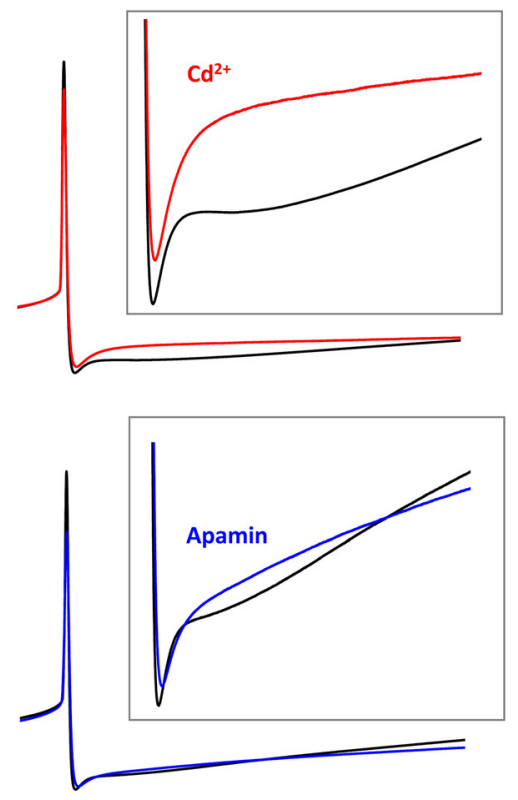

E
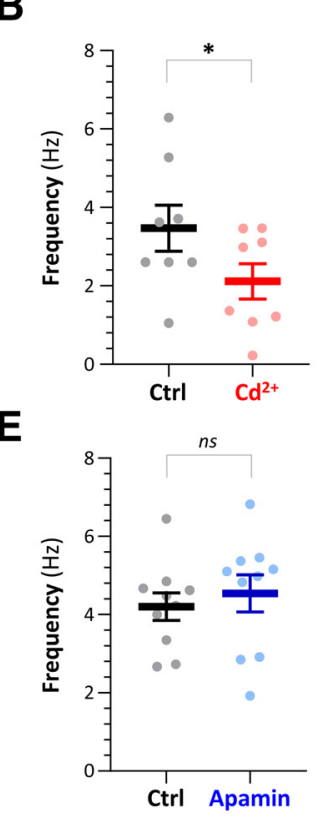

H
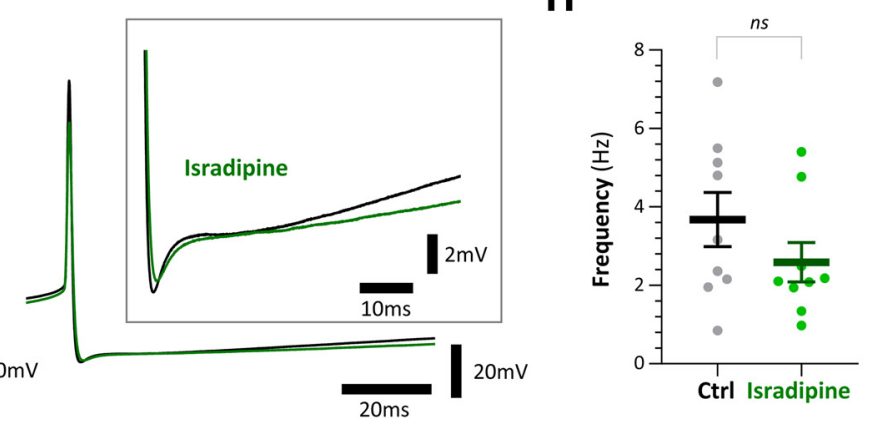

C

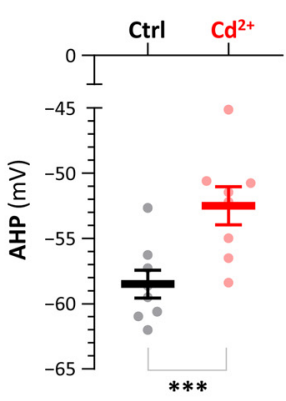

F

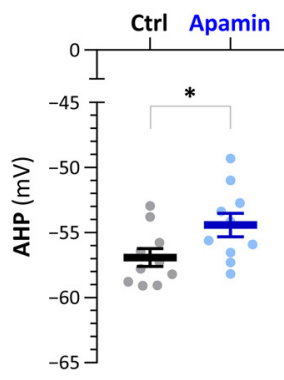

I

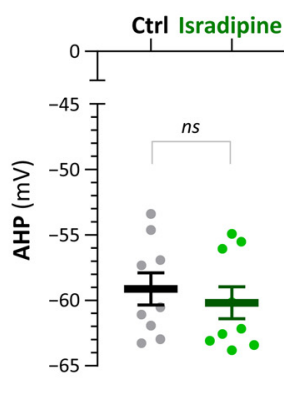

Figure 6. Role of calcium in modulating pacemaker activity in zebrafish DC2/4 dopaminergic neurons. Pacemaker activity in dopaminergic neurons was recorded in control solution (control; black) and after addition of: $(\boldsymbol{A}-\boldsymbol{C}) \mathrm{Cd}^{2+}$ (voltage-gated calcium channel blocker; final bath concentration $50 \mu \mathrm{m} ; n=8$ cells; red), (D-F) apamin (SK channel blocker; final bath concentration $100 \mathrm{~nm} ; n=10$ cells; blue), or (G-I) isradipine (L-type calcium channel blocker; final bath concentration $9 \mu \mathrm{m} ; n=9$ cells; green). A, D, G, Membrane potential traces showing pacemaker activity under control conditions and following addition of each inhibitor. In each case, averaged APs are shown to the right of the trace; the inset panels show the AHP using a larger scale to illustrate differences. $\boldsymbol{B}, \boldsymbol{E}, \boldsymbol{H}$, Scatter plots showing discharge frequency of spontaneous pacemaker activity under control conditions and after addition of each inhibitor. Points show data from individual cells, bars show mean $\pm \mathrm{SE} ; * p<0.05$ (two-tailed paired $t$ test). $C, F, I$, Scatter plots showing the amplitude of the slow AHP under control conditions and after addition of each inhibitor. Points show data from individual cells, bars show mean $\pm \mathrm{SE} ; * p<0.05, * * * p<0.001$ (two-tailed paired $t$ test). ns, not significant.

changes in AHP do not account for the observed increase in pacemaker frequency in calcium-free conditions. Hence, increase of the firing frequency in calcium-free medium is produced by the action of extracellular $\mathrm{Ca}^{2+}$ ions on sodium channel gating (Armstrong and Cota, 1991).

In rodent dopaminergic neurons, small-conductance calcium-sensitive potassium channels (SK channels) and L-type calcium channels are implicated in autonomous firing (Ping and Shepard, 1996; Wolfart et al., 2001; Chan et al., 2007; Puopolo et al., 2007; Putzier et al., 2009). We tested these mechanisms in zebrafish dopaminergic neurons. Application of the SK-channel blocker apamin $(100 \mathrm{~nm})$ resulted in a small $(\approx 2.5 \mathrm{mV})$ but statistically significant reduction in the slow AHP (baseline $-56.9 \pm 2.2 \mathrm{mV}$ vs apamin $-54.4 \pm 2.8 \mathrm{mV} ; n=10 ; p<0.05)$. Apamin did not influence the fast AHP (baseline $-64.4 \pm 2.2 \mathrm{mV}$ vs apamin $-62.5 \pm 2.8 \mathrm{mV}$ ), threshold potential (baseline $-36.5 \pm$ $3.0 \mathrm{mV}$ vs apamin $-36.4 \pm 2.5 \mathrm{mV}$ ), or frequency of autonomous firing (baseline $4.2 \pm 1.1 \mathrm{~Hz}$ vs apamin $4.5 \pm 1.5 \mathrm{~Hz}$; Fig. $6 D-F$ ). Blockade of L-type calcium channels with $9 \mu \mathrm{M}$ isradipine did not affect AHP or threshold potential, but caused a non-significant trend toward reduced firing frequency (Fig. $6 G-I$ ). This suggests that L-type channels are not involved in the generation or regulation of autonomous activity in zebrafish dopaminergic neurons.

Together, these data show that intracellular calcium can alter pacemaker frequency by modulating the AHP, likely by affecting calcium-sensitive potassium channels. Conversely, extracellular calcium likely influences AP threshold through a direct effect on sodium channels. Consequently, while not directly involved in generating pacemaker activity, calcium can modulate autonomous activity in zebrafish DC2/4 dopaminergic neurons through actions on sodium and potassium channels.

To summarize, the data presented so far show that voltagegated sodium channels $\left(\mathrm{Na}_{\mathrm{V}}\right)$ are a critical mediator of the inward current that causes spontaneous depolarization of zebrafish dopaminergic neurons, thereby driving pacemaker activity. Under sodium channel blockade with TTX, no oscillations of membrane potential were observed, either at rest or in response to injection of depolarizing or hyperpolarizing current steps. Furthermore, pacemaker activity in zebrafish dopaminergic neurons was not abolished by blockade of ionic channels that mediate pacemaker activity in other diverse types of neurons, such as HCN channels, SK channels, calcium channels and calcium- 
A

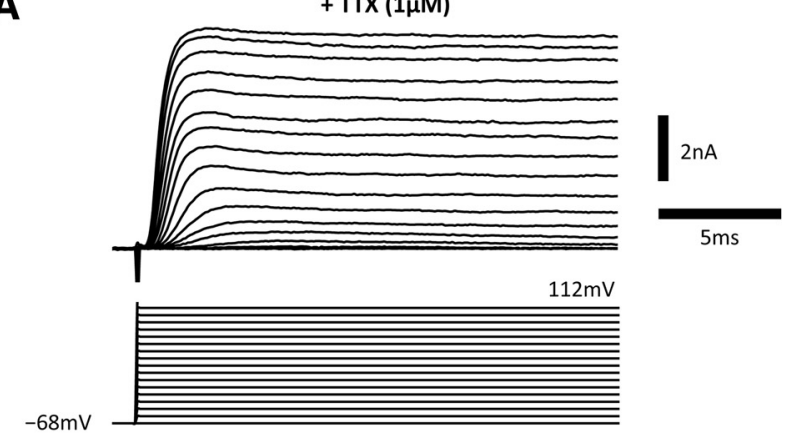

B

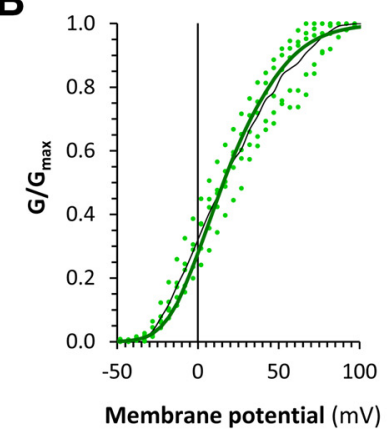

C

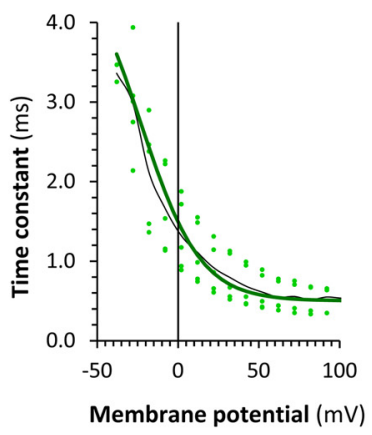

D

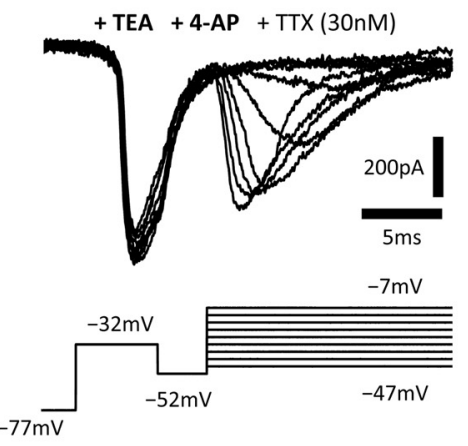

E + TEA + 4-AP + TTX (30nM)

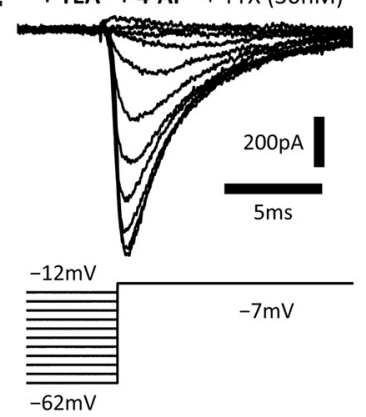

$\mathbf{F}$

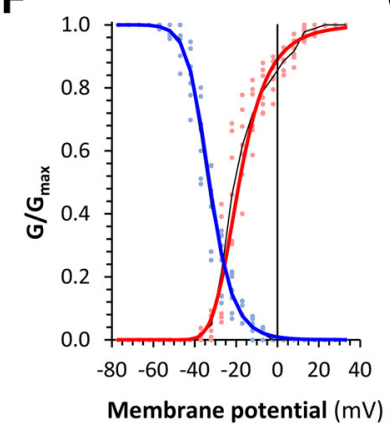

G

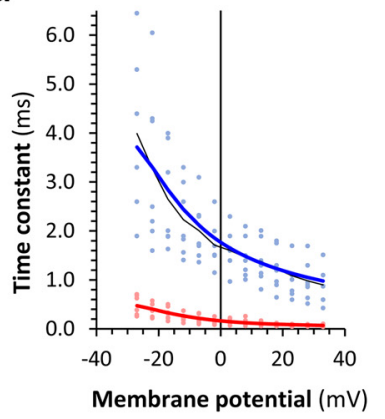

Figure 7. Activation and inactivation of sodium and potassium currents in zebrafish $D C 2 / 4$ dopaminergic neurons. $\boldsymbol{A}$, The traces show a family of potassium currents evoked in a dopaminergic neuron by holding potential steps between -68 and $112 \mathrm{mV}$ in 10-mV increments as indicated below. Whole-cell voltage clamp recordings were made in the presence of TTX (1 $\mu \mathrm{M}$ ) to block sodium channels. $\boldsymbol{B}$, Activation curve for voltage-gated potassium channels. C, Dependence of time constant of activation of voltage gated potassium channels on membrane potential. $\boldsymbol{D}$, The traces show a family of sodium currents evoked in a dopaminergic neuron by holding potential steps between $-47 \mathrm{and}-7 \mathrm{mV}$ in 5 -mV increments, following a prepulse protocol (sequential steps to $-77,-32$, and $-52 \mathrm{mV}$ as shown in the lower tracing), to measure sodium channel activation. The prepulse protocol and addition of low-concentration TTX (30 nM) were necessary to ensure space clamp during whole-cell recording. Potassium channels were blocked by adding TEA (20 mM) and 4-AP (2 mM) to the extracellular solution, and replacing potassium with cesium in the recording electrode. $\boldsymbol{E}$, The traces show a family of sodium currents evoked by a holding potential step to $-7 \mathrm{mV}$ from holding potentials between -62 and $-12 \mathrm{mV}$ in $5-\mathrm{mV}$ increments, to measure sodium channel inactivation. Data are from the same cell as in panel $\boldsymbol{D}$, recorded under identical experimental conditions. $\boldsymbol{F}$, Activation (red) and inactivation (blue) curves for voltage-gated sodium channels. G, Dependence of time constant of activation (red) and inactivation (blue) of voltage gated sodium channels on membrane potential. In panels $B, C, F, G$, colored data points show measurements from individual cells (potassium currents $n=5$ cells, 5 slices from 3 brains; sodium currents, $n=7$ cells, 7 slices from 5 brains), and the thin black line shows the averaged experimental data. The superimposed colored lines show functions derived from fitting Hodgkin-Huxley model equations to the experimental data (see Materials and Methods; Eqs. 6-29).

dependent conductances (although blockade of some these channels modulated the frequency of AP discharge). Together, these data suggest that sodium channels mediate the inward current that is necessary for spontaneous activity. In addition, the low density of potassium leak channels resulted in a relatively depolarized resting membrane potential that may be close to values at which voltage-gated sodium channels start to become activated. Finally, voltage-gated potassium channels mediating the AHP are necessary for fast recovery of sodium channels from inactivation and preparing them for generation of the next AP.

These experimental data therefore suggest that interaction of just three components is critical for autonomous pacemaker activity in zebrafish DC2/4 dopaminergic neurons: voltage-gated sodium and potassium conductances, and a leak conductance. To test this hypothesis in a computer model simulation, we next determined experimentally the voltage dependence and kinetics of activation and inactivation of sodium and potassium currents in zebrafish DC2/4 dopaminergic neurons.

\section{Measurement of activation and inactivation of sodium and} potassium currents in zebrafish dopaminergic neurons

Potassium currents were isolated using $1 \mu \mathrm{M}$ TTX to block sodium channels, and recorded during a series of holding potential steps from -68 to $112 \mathrm{mV}$ (Fig. 7A). After compensation for leak currents, we measured the peak amplitude of each response and calculated peak conductance (see Materials and Methods; Eq. 1), which was normalized, and plotted against membrane potential (Fig. 7B). We further determined the time constant of each response onset by fitting the data to an exponential function (Eq. 2). Time constants were then plotted against membrane potential (Fig. 7C).

Sodium currents were isolated using $20 \mathrm{~mm}$ TEA and $2 \mathrm{~mm} 4-$ AP in the extracellular solution, and cesium-based intracellular solution, to block potassium currents. To achieve space clamp during measurement of sodium channel properties, low-concentration (30 nм) TTX was also added to the extracellular solution and activation of sodium currents was measured using a stimulation protocol that included a prepulse to selectively inactivate axonal sodium channels (Milescu et al., 2010; see Materials and Methods, Measurement of voltage-gated potassium and sodium channel dynamics). Figure $7 D$ shows the sodium current of a dopaminergic neuron recorded using this approach, after subtraction of leak currents. The peak current for each response was measured, allowing calculation of conductance (Eq. 3), which was normalized to the maximum and plotted against membrane potential (Fig. $7 F$, red). Activation time constants were calculated by fitting the data from the beginning to the peak of the current responses to an exponential function (Eq. 4; Fig. 7G, red). Sodium current inactivation was measured by clamping the membrane potential at $-7 \mathrm{mV}$ for $20 \mathrm{~ms}$, after holding it for 
$50 \mathrm{~ms}$ at values between -62 and $-12 \mathrm{mV}$, in $5-\mathrm{mV}$ increments (Fig. $7 E$ ). Current responses were used to calculate conductance, from which the inactivation curve for sodium channels was derived (Fig. $7 F$, blue). Inactivation time constants were calculated using an exponential fit function (Eq. 5) from the peak response to the end (Fig. $7 G$, blue).

We next used Hodgkin-Huxley formalism to fit model equations to these experimental observations. The mathematical procedures employed to accomplish this are detailed in Materials and Methods (Eqs. 6-29), parameters describing channel behavior (derived by fitting model equations to the experimental data) are shown in Table 1. Curves for conductance versus membrane potential (Fig. $7 B, F$ ) and time constant versus membrane potential (Fig. $7 C, G$ ), employing these parameters, were superimposed on the observed experimental measurements of the same properties. The derived functions showed close alignment with the experimental data, suggesting that the calculated parameters are valid for use in a computational simulation.

\section{Computational model of a zebrafish dopaminergic neuron}

To test whether sodium and potassium conductances with the properties measured in zebrafish dopaminergic neurons are sufficient to account for pacemaker activity, we employed a computational approach. The model has a single compartment with the three conductances identified by experimental observations in zebrafish DC2/4 dopaminergic neurons: a leak conductance, voltage-gated sodium channels, and voltage-gated potassium channels. The soma diameter was set at $14.5 \mu \mathrm{m}$, and the length $35 \mu \mathrm{m}$, corresponding to the measured dimensions of the recorded cells. Membrane capacitance was set to $5 \mu \mathrm{F} / \mathrm{cm}^{2}$ and leak conductance $0.1 \mathrm{mS} / \mathrm{cm}^{2}$, allowing the model to replicate experimental measurements for cell membrane capacitance and resistance. The reversal potential for the leak current was set to $-43.5 \mathrm{mV}$, corresponding to the resting membrane potential of experimentally-recorded cells whose pacemaker activity was inactivated by TTX. The density of sodium channels was set to $23 \mathrm{mS} / \mathrm{cm}^{2}$, so that the amplitude and overshoot of APs in the model corresponded to the experimentally-measured values. Potassium channel density was set to $15 \mathrm{mS} / \mathrm{cm}^{2}$.

Using these parameters, the model cell generated autonomous pacemaker activity at a frequency of $3.5 \mathrm{~Hz}$ (Fig. $8 \mathrm{~A}$, black), corresponding closely to the observed spontaneous firing frequency of dopaminergic neurons $(3.1 \pm 1.5 \mathrm{~Hz})$. Since the aim of this model was to test whether pacemaker activity can be replicated by the interaction of voltage-gated sodium and potassium channels, the model did not include voltage-gated calcium channels or calcium-sensitive potassium channels. Consequently, the waveform of the model-generated APs most closely resembled experimental recordings made in extracellular solution containing cadmium to block calcium channels (Fig. 8A, red). The model also replicated the experimentally-recorded responses of dopaminergic neurons to injection of depolarizing and hyperpolarizing current (Fig. 8B). This included the dependence of firing frequency on the amplitude of injected current, depolarizing block after injection of large currents, and suppression of activity during injection of hyperpolarizing currents (Fig. 8B,C).

Next, we used the computational model to explore how changes in ionic conductances affect pacemaker properties. Changing any of the three conductances, while keeping the other two constant, altered the firing frequency of the simulated neuron. Frequency increased with leak conductance, from $0 \mathrm{~Hz}$ at $0.085 \mathrm{mS} / \mathrm{cm}^{2}$ to a maximum of $28 \mathrm{~Hz}$ at $1.2 \mathrm{mS} / \mathrm{cm}^{2}$. Further increases in leak conductance beyond this value caused a decrease in firing frequency; pacemaker activity ceased at values above $2 \mathrm{mS} / \mathrm{cm}^{2}$ (Fig. $8 D$ ). The model generated autonomous activity with voltage-gated potassium conductance in the range $8.0-15.9 \mathrm{mS} / \mathrm{cm}^{2}$ (Fig. $8 E$ ). Increasing the potassium conductance from $15 \mathrm{mS} / \mathrm{cm}^{2}$ led to a decrease in the frequency of the simulated pacemaker, which stopped completely above $>16 \mathrm{mS} /$ $\mathrm{cm}^{2}$. Conversely, decreasing the potassium conductance between 15 and $10 \mathrm{mS} / \mathrm{cm}^{2}$ led to a gradual increase in pacemaker frequency to $\sim 10 \mathrm{~Hz}$; further decreases of the potassium conductance resulted in a dramatic increase in frequency and then loss of activity (Fig. 8E). Sodium conductances between 21.1-41.0 mS/ $\mathrm{cm}^{2}$ were accompanied by spontaneous activity; the discharge frequency increased with conductance to $18 \mathrm{~Hz}$ at $41 \mathrm{mS} / \mathrm{cm}^{2}$. Between 41 and $44 \mathrm{mS} / \mathrm{cm}^{2}$, the simulated neuron fired bursts instead of single APs, and at conductances above $45 \mathrm{mS} / \mathrm{cm}^{2}$ autonomous activity was lost.

Overall, these computational simulations show that three ionic conductances (leak current, voltage-gated sodium channels, and voltage-gated potassium channels), whose properties replicate our experimental observations, are sufficient to drive spontaneous rhythmic discharges in a model neuron. Simulation results also show that the proposed pacemaker mechanism is robust, because the model generated spontaneous APs while any of the three conductances varied over a broad range. At the same time, all three conductances were necessary for spontaneous firing: eliminating any one of them (or reducing it below a minimal value) abolished spontaneous firing in the model.

The dynamic interactions of voltage-gated sodium, voltagegated potassium, and leak currents that cause the rhythmic generation of APs in the model are illustrated in Figure 9. The upstroke of the AP is mediated by voltage-gated sodium channels, which are inactivated by the resulting strong depolarization. Together with activation of voltage-gated potassium channels, this causes the membrane potential to become hyperpolarized to values below $-60 \mathrm{mV}$. At these potentials, current flowing through voltage-gated sodium and potassium channels is negligible; consequently, the leak current dominates the net inward current at the start of the interspike interval, driving the membrane potential toward the leak current reversal potential of $-43.5 \mathrm{mV}$. This depolarization progressively activates voltage-gated sodium and potassium channels. The steeper activation curve for sodium channels and stronger electrochemical gradient for sodium ions ensure (in combination with the leak current) that a net inward current is maintained throughout the interspike interval, causing gradual but relentless depolarization. Well before the leak current reversal potential is reached, a large and rapidly increasing sodium current dominates the net inward current. This results in regenerative activation of voltage-gated sodium channels that initiates the next AP.

We conclude that zebrafish DC2/4 dopaminergic neurons generate robust autonomous activity using a simple pacemaker mechanism that relies on a minimal set of ionic conductances, mediated by voltage-gated sodium and potassium channels and leak channels. While the minimal configuration of the three conductances is both necessary and sufficient for generation of rhythmic spiking, the frequency of spontaneous firing in zebrafish DA neurons also depends on the slow AHP component. Potassium currents mediating slow AHP contribute to the rate of membrane depolarization during the interspike interval, so that modulation of these currents can change the firing frequency of zebrafish DC2/4 dopaminergic neurons. 
A

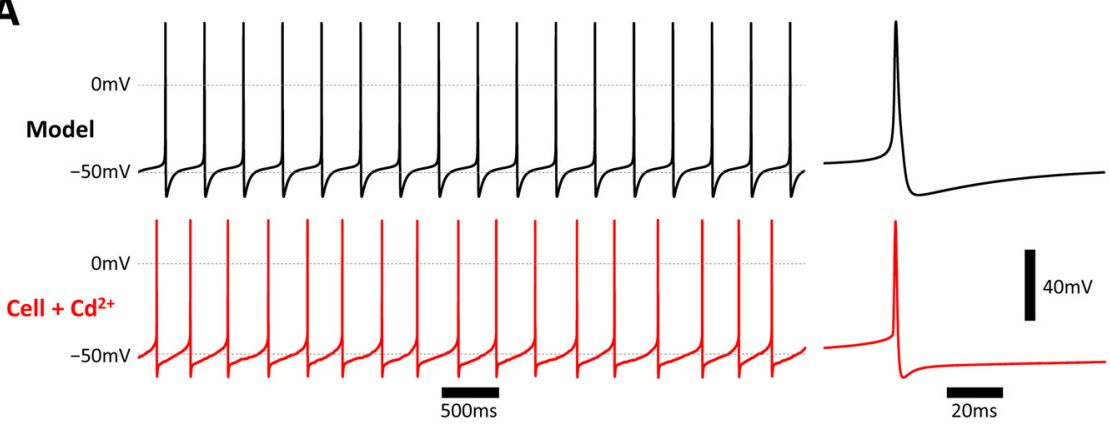

B

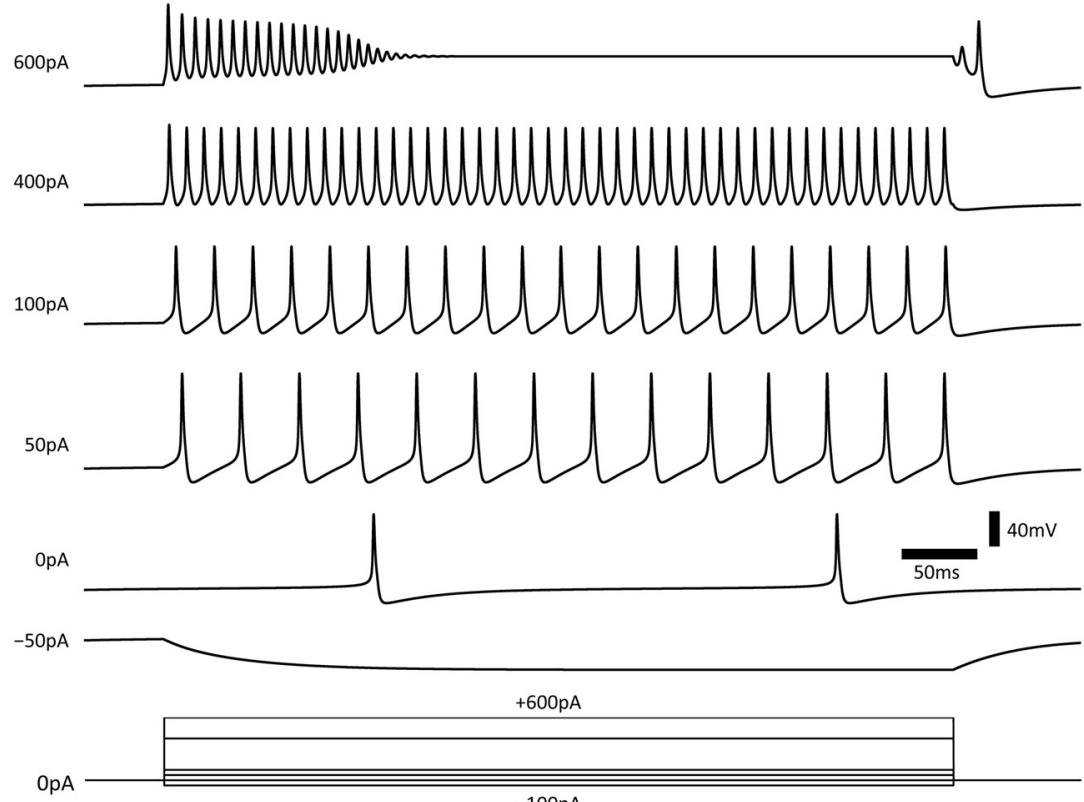

C

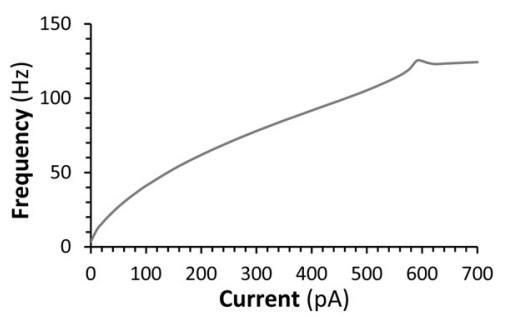

D

E
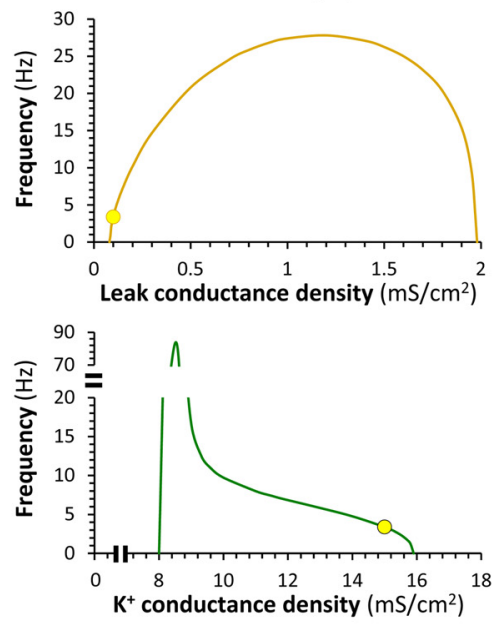

$\mathbf{F}$

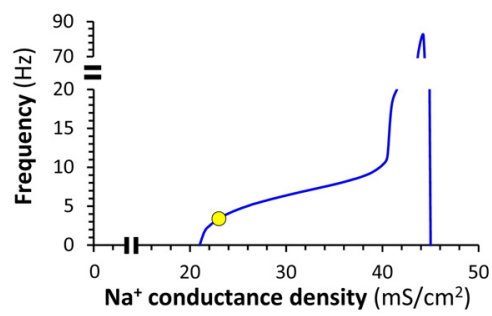

Figure 8. Computational model of a zebrafish DC2/4 dopaminergic neuron replicates autonomous pacemaker activity. $A$, Autonomous pacemaker activity (left) and average AP (right) for a computational simulation of a dopaminergic neuron (upper trace; black) compared with an electrophysiological recording from a zebrafish dopaminergic neuron in extracellular solution with $50 \mu \mathrm{m}$ cadmium to block calcium channels (lower trace; red). $B$, Membrane potential responses of the model neuron to injection of current steps with amplitude from -50 to $+600 \mathrm{pA}$, as indicated in the lower traces. $\boldsymbol{C}$, Dependence of the firing frequency of the model neuron on the amplitude of the injected current steps. $\boldsymbol{D}$, Relationship between the autonomous firing frequency of the model neuron and the leak conductance density. $\boldsymbol{E}$, Relationship between the autonomous firing frequency of the model neuron and the voltage-gated potassium conductance density. $\boldsymbol{F}$, Relationship between the autonomous firing frequency of the model neuron and the voltage-gated sodium conductance density. In panels $\boldsymbol{D}-\boldsymbol{F}$, the experimentally-determined values used in the simulations shown in panels $\boldsymbol{A}-\mathrm{C}$ are marked with yellow symbols.

\section{Discussion}

We developed a brain slice preparation from adult zebrafish using a novel transgenic $\operatorname{Tg}($ th:gal4 $)$; $\operatorname{Tg}(U A S: e g f p)$ line to investigate the electrophysiology of DC2/4 dopaminergic neurons by whole-cell patch clamp recordings. Prior work using loose and perforated patch recordings from DC2 neurons of larval zebrafish (Jay et al., 2015) reported rhythmic discharges suggestive of pacemaker activity at $1.5-2 \mathrm{~Hz}$ independent of synaptic inputs, and short bursts up to $23 \mathrm{~Hz}$ driven by synaptic inputs. Our results are compatible with recordings in larvae and significantly extend prior work, by (1) demonstrating that DC2/4 neurons share many basic electrophysiological properties with dopaminergic neuronal populations from other species; and (2) providing insights into mechanisms of pacemaker activity of DC2/4 neurons. Both zebrafish and mammalian dopaminergic neurons show cell-intrinsic, autonomous firing. However, the pacemaker mechanism in zebrafish DC2/4 neurons differs significantly from the pacemakers of mammalian dopaminergic neurons. Our results contribute to understanding the comparative physiology of the dopaminergic system and are important because they suggest opportunities to address the contributions of pacemaker mechanisms to the selective susceptibility of dopaminergic neurons in the pathogenesis of PD.

\section{The electrophysiology of zebrafish DC2/4 cells}

The relatively depolarized resting membrane potential of zebrafish DC2/4 dopaminergic neurons was near to the AP threshold, facilitating autonomous firing. Blocking voltage-gated sodium channels with TTX prevented APs and also caused depolarization relative to the interspike potential. This suggests that activity-dependent hyperpolarizing mechanisms, such as activation of potassium channels, contribute significantly to the interspike membrane potential. Consequently, when TTX blocks spiking, potassium channels are not activated, leading to decreased net potassium current and depolarization. Notably, removal of sodium from the extracellular solution under TTX blockade did not change the membrane potential further, indicating that sodium leak conductance does not contribute significantly to the depolarized resting potential. This differs from mammalian VTA neurons, in which sodium leak conductance is an important contributor to the depolarized resting membrane potential (Khaliq and Bean, 2010). Removing extracellular calcium during TTX 


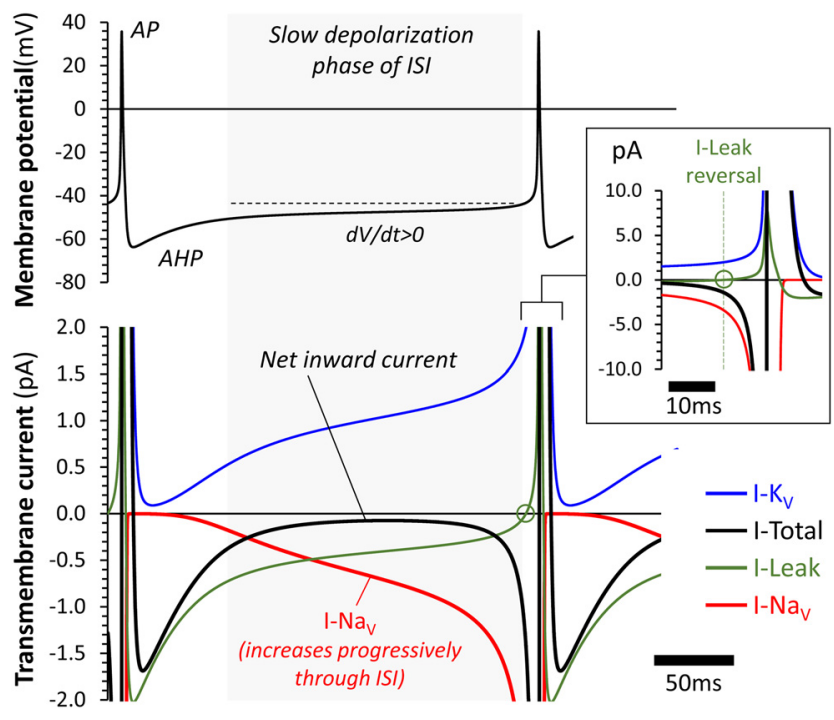

Figure 9. Ionic currents underlying pacemaker activity in a computational model of a zebrafish DC2/4 dopaminergic neuron. The upper panel shows the membrane potential of the model cell, from the start of one AP to the conclusion of the next. The slow depolarization phase of the interspike interval is indicated and shaded for clarity. The lower panel shows currents mediated by voltage-gated sodium channels (red), voltage gated potassium channels (blue) and leak conductance (green), along with the net current (black). The inset panel shows the period immediately before the AP, with expanded time scale and compressed current scale to illustrate the large and rapidly increasing current mediated by voltage-gated sodium channels as the leak current reversal potential (circled) is passed.

blockade also did not alter the membrane potential of zebrafish DC2/4 neurons, arguing against a significant contribution of calcium conductance to the resting potential. Instead, we propose that the depolarized resting membrane potential of zebrafish dopaminergic neurons is attributable to a low density of potassium leak channels. This interpretation is consistent both with our observation that the input resistance of these neurons is high, and with prior studies reporting low expression levels of potassium leak channels in dopaminergic neurons from other species (Karschin et al., 1996; Talley et al., 2001).

Zebrafish DC2/4 neurons showed broad APs with pronounced AHP, similar to the APs reported in midbrain dopaminergic neurons from rats, mice and guinea pigs (Grace and Onn, 1989; Yung et al., 1991; Häusser et al., 1995; Khaliq and Bean, 2010). The shape, amplitude and duration of APs recorded in zebrafish DC2/4 dopaminergic neurons did not change after removal of extracellular calcium, or during blockade of calcium channels with cadmium. This suggests that, in contrast to mammalian SNc neurons (Yung et al., 1991), APs in zebrafish DC2/4 neurons lack a significant calcium-mediated component. In this respect, they resemble mammalian VTA dopaminergic neurons (Khaliq and Bean, 2010).

\section{Mechanism of pacemaker activity in zebrafish DA neurons}

Zebrafish DC2/4 dopaminergic neurons discharged autonomously, rhythmically, and robustly at $2-4 \mathrm{~Hz}$. In response to depolarizing current, firing rates could reach up to $50-70 \mathrm{~Hz}$ with little adaptation. In contrast, mouse, rat or guinea pig midbrain dopaminergic neurons, which show similar autonomous discharge frequencies, can only be driven to sustained firing at 10-15 Hz (Yung et al., 1991; Richards et al., 1997), or short bursts of up to $30 \mathrm{~Hz}$ (Blythe et al., 2009). The ability to sustain highfrequency firing may be related to the direct caudal projections of DC2/4 neurons to spinal cord motor circuits (Tay et al., 2011) and suggests that the underlying pacemaker employs elements with rapid kinetics, such as sodium and potassium channels. Our experimental observations support this prediction. With sodium channels blocked by TTX, we did not observe calcium-mediated subthreshold oscillations, which are centrally involved in the pacemaker activity of mammalian SNc neurons (Yung et al., 1991; Ping and Shepard, 1996; Chan et al., 2007; Putzier et al., 2009). Furthermore, blockade of L-type calcium channels, an essential component of the adult mouse SNc pacemaker (Chan et al., 2007; Putzier et al., 2009), or removal of calcium from the extracellular medium, did not abrogate autonomous firing in zebrafish DC2/4 neurons. Similar to mouse VTA neurons (Khaliq and Bean, 2010), firing frequency increased in the absence of extracellular calcium, likely attributable to a hyperpolarizing shift in the activation of sodium channels (Armstrong and Cota, 1991). Together, these observations indicate that the pacemaker in zebrafish DC2/4 dopaminergic neurons is calciumindependent.

We also found that several other mechanisms implicated in mammalian dopaminergic neuronal pacemakers are not essential for autonomous firing of zebrafish DC2/4 neurons. HCN cation channels are essential for pacemaker activity in diverse CNS neurons and the heart (Pape, 1996; Surmeier et al., 2005). HCN channels are expressed in mammalian dopaminergic neurons (Yung et al., 1991) and are involved in pacemaker activity in juvenile wildtype (Neuhoff et al., 2002) and adult $\mathrm{Ca}_{\mathrm{V}} 1.3^{-/-}$and isradipineexposed (Chan et al., 2007) mouse SNc neurons, which (in contrast to adult wild-type SNc neurons) do not rely on calcium currents for autonomous activity. However, similar to mammalian VTA neurons (Neuhoff et al., 2002), application of the HCN antagonist ZD7288 did not alter pacemaker activity in zebrafish DC2/4 neurons and we found no evidence of membrane potential sag indicating an $\mathrm{I}_{\mathrm{h}}$ current during hyperpolarizing pulses. Furthermore, calcium-dependent potassium conductances, including SK channels thought critical for pacemaker firing in mammalian SNc neurons (Ping and Shepard, 1996; Wolfart et al., 2001; Wolfart and Roeper, 2002), were not necessary for pacemaker activity in zebrafish DC2/4 neurons, although they did influence AHP.

These data, supported by computational simulations using experimentally-derived conductance measurements, show that only three elements, a small leak conductance, voltage-gated sodium channels and voltage-gated potassium channels, are necessary for autonomous firing of zebrafish DC2/4 neurons. This is significantly simpler than in rodent neurons, where pacemaker mechanisms include calcium oscillations in SNc and non-voltage-dependent sodium currents in VTA, suggesting that the more complex features of the mammalian pacemakers are a recent evolutionary development.

\section{Regulation by synaptic inputs}

Current injection could modulate or suspend pacemaker activity in DC2/4 neurons, suggesting that synaptic inputs may regulate intrinsic activity. This is compatible with prior work showing changes in the firing frequency of larval DC2 neurons that were dependent on synaptic function (Jay et al., 2015). Calcium-imaging studies in transgenic GCaMP7a zebrafish larvae suggested that episodes of spontaneous and stimulus-evoked neural activity correlated strongly between dopaminergic neurons (Reinig et al., 2017). We predict that synchronous periods of activity and inactivity within neuronal ensembles could be driven by shared synaptic inputs that modulate cell-intrinsic pacemakers. However, studies using a preparation that preserves synaptic inputs will be necessary to investigate this possibility. 


\section{Implications for using zebrafish models to study PD}

It is important to understand the extent to which model systems replicate human disease, since this has important consequences for the applicability of experimentally-derived insights to pathophysiology. In the mouse $\mathrm{SNc}$, pharmacological blockade of $\mathrm{Ca}_{\mathrm{V}} 1.3$ channels mediating pacemaker currents prevented toxicant-induced pathology and was proposed as a neuroprotective intervention in PD (Chan et al., 2007). However, a Phase III clinical trial (Simuni, 2020) did not find evidence that isradipine prevented the progression of $\mathrm{PD}$, so the contribution of L-type channel-mediated calcium currents to pathophysiology remains uncertain. The absence of a $\mathrm{Ca}_{\mathrm{V}} 1.3$-dependent pacemaker in zebrafish DC2/4 dopaminergic neurons may provide opportunities to resolve this question experimentally, for example, by testing whether transgenic expression of $\mathrm{Ca}_{\mathrm{V}} 1.3$ channel subunits will modulate the susceptibility of zebrafish dopaminergic neurons to genetic or environmental etiologic triggers relevant to PD. Furthermore, the calcium-independent pacemaker of zebrafish DC2/4 neurons may allow the pathogenic role of sustained autonomous firing to be dissociated from the ionic fluxes that drive the pacemaker. Regardless, cellular and molecular insights gained into PD pathogenesis using both genetic (Flinn et al., 2013; O'Donnell et al., 2014; Soman et al., 2017; Zhang et al., 2017) and toxicant (Dukes et al., 2016; Lulla et al., 2016) models suggest the lack of a $\mathrm{Ca}_{\mathrm{V}} 1.3$-mediated pacemaker current in DC2/4 neurons is not a serious limitation to the use of zebrafish to study PD. However, it is important to note that the absence of a calcium-dependent pacemaker and lack of a significant contribution of calcium currents to the AP do not imply that calcium plays no part in the physiology or pathophysiology of zebrafish DC2/4 neurons. Activity-dependent changes in cytosolic calcium have been demonstrated in zebrafish dopaminergic neurons by imaging (Reinig et al., 2017), our data strongly implicate calcium in regulating the AHP of DC2/4 neurons through calcium-sensitive potassium channels, and published studies show that intracellular calcium dynamics are critically important in mediating loss of dopaminergic neurons in zebrafish lacking the PD-related protein Pink1 (Soman et al., 2017).

\section{References}

Armstrong CM, Cota G (1991) Calcium-ion as a cofactor in Na channel gating. Proc Natl Acad Sci USA 88:6528-6531.

Bai Q, Parris RS, Burton EA (2014) Different mechanisms regulate expression of zebrafish myelin protein zero (P0) in myelinating oligodendrocytes and its induction following axonal injury. J Biol Chem 289:2411424128.

Banerjee K, Sinha M, Pham CLL, Jana S, Chanda D, Cappai R, Chakrabarti S (2010) Alpha-synuclein induced membrane depolarization and loss of phosphorylation capacity of isolated rat brain mitochondria: implications in Parkinson's disease. FEBS Lett 584:1571-1576.

Baranauskas G (2007) Ionic channel function in action potential generation: current perspective. Mol Neurobiol 35:129-150.

Bartel WP, Van Laar VS, Burton EA (2020) Parkinson's disease. In: Behavioral and neural genetics of zebrafish (Gerlai RT, ed), pp 377-412. San Diego: Elsevier.

Blythe SN, Wokosin D, Atherton JF, Bevan MD (2009) Cellular mechanisms underlying burst firing in substantia nigra dopamine neurons. J Neurosci 29:15531-15541.

Braak H, Del Tredici K (2004) Poor and protracted myelination as a contributory factor to neurodegenerative disorders. Neurobiol Aging 25:19-23.

Chan CS, Guzman JN, Ilijic E, Mercer JN, Rick C, Tkatch T, Meredith GE, Surmeier DJ (2007) 'Rejuvenation' protects neurons in mouse models of Parkinson's disease. Nature 447:1081-1086.

Chistiakova M, Ilin V, Roshchin M, Bannon N, Malyshev A, Kisvárday Z, Volgushev M (2019) Distinct heterosynaptic plasticity in fast spiking and non-fast-spiking inhibitory neurons in rat visual cortex. J Neurosci 39:6865-6878.

Desplats P, Lee HJ, Bae EJ, Patrick C, Rockenstein E, Crews L, Spencer B, Masliah E, Lee SJ (2009) Inclusion formation and neuronal cell death through neuron-to-neuron transmission of alpha-synuclein. Proc Natl Acad Sci USA 106:13010-13015.

Devi L, Raghavendran V, Prabhu BM, Avadhani NG, Anandatheerthavarada HK (2008) Mitochondrial import and accumulation of alpha-synuclein impair complex I in human dopaminergic neuronal cultures and Parkinson disease brain. J Biol Chem 283:9089-9100.

Di Maio R, Barrett PJ, Hoffman EK, Barrett CW, Zharikov A, Borah A, Hu X, McCoy J, Chu CT, Burton EA, Hastings TG, Greenamyre JT (2016) $\alpha$-Synuclein binds to TOM20 and inhibits mitochondrial protein import in Parkinson's disease. Sci Transl Med 8:342ra78.

Dukes AA, Bai Q, Van Laar VS, Zhou Y, Ilin V, David CN, Agim ZS, Bonkowsky JL, Cannon JR, Watkins SC, Croix CM, Burton EA, Berman SB (2016) Live imaging of mitochondrial dynamics in CNS dopaminergic neurons in vivo demonstrates early reversal of mitochondrial transport following MPP $(+)$ exposure. Neurobiol Dis 95:238-249.

Flinn LJ, Keatinge M, Bretaud S, Mortiboys H, Matsui H, De Felice E, Woodroof HI, Brown L, McTighe A, Soellner R, Allen CE, Heath PR, Milo M, Muqit MM, Reichert AS, Köster RW, Ingham PW, Bandmann O (2013) TigarB causes mitochondrial dysfunction and neuronal loss in PINK1 deficiency. Ann Neurol 74:837-847.

Grace AA, Bunney BS (1983) Intracellular and extracellular electrophysiology of nigral dopaminergic neurons-1. Identification and characterization. Neuroscience 10:301-315.

Grace AA, Onn SP (1989) Morphology and electrophysiological properties of immunocytochemically identified rat dopamine neurons recorded in vitro. J Neurosci 9:3463-3481.

Hastings TG (2009) The role of dopamine oxidation in mitochondrial dysfunction: implications for Parkinson's disease. J Bioenerg Biomembr 41:469-472.

Hauser DN, Dukes AA, Mortimer AD, Hastings TG (2013) Dopamine quinone modifies and decreases the abundance of the mitochondrial selenoprotein glutathione peroxidase 4. Free Radic Biol Med 65:419-427.

Häusser M, Stuart G, Racca C, Sakmann B (1995) Axonal initiation and active dendritic propagation of action-potentials in substantia-nigra neurons. Neuron 15:637-647.

Hines ML, Carnevale NT (1997) The NEURON simulation environment. Neural Comput 9:1179-1209.

Hodgkin AL, Huxley AF (1952) A quantitative description of membrane current and its application to conduction and excitation in nerve. J Physiol 117:500-544.

Hsu LJ, Sagara Y, Arroyo A, Rockenstein E, Sisk A, Mallory M, Wong J, Takenouchi T, Hashimoto M, Masliah E (2000) alpha-synuclein promotes mitochondrial deficit and oxidative stress. Am J Pathol 157:401410.

Jay M, De Faveri F, McDearmid JR (2015) Firing dynamics and modulatory actions of supraspinal dopaminergic neurons during zebrafish locomotor behavior. Curr Biol 25:435-444

Karschin C, Dissmann E, Stühmer W, Karschin A (1996) IRK(1-3) and GIRK(1-4) inwardly rectifying $\mathrm{K}+$ channel mRNAs are differentially expressed in the adult rat brain. J Neurosci 16:3559-3570.

Khaliq ZM, Bean BP (2010) Pacemaking in dopaminergic ventral tegmental area neurons: depolarizing drive from background and voltage-dependent sodium conductances. J Neurosci 30:7401-7413.

Kim SH, Choi YM, Jang JY, Chung S, Kang YK, Park MK (2007) Nonselective cation channels are essential for maintaining intracellular $\mathrm{Ca} 2+$ levels and spontaneous firing activity in the midbrain dopamine neurons. Pflugers Arch 455:309-321.

Luk KC, Song C, O'Brien P, Stieber A, Branch JR, Brunden KR, Trojanowski JQ, Lee VM (2009) Exogenous alpha-synuclein fibrils seed the formation of Lewy body-like intracellular inclusions in cultured cells. Proc Natl Acad Sci USA 106:20051-20056.

Lulla A, Barnhill L, Bitan G, Ivanova MI, Nguyen B, O’Donnell K, Stahl MC, Yamashiro C, Klärner FG, Schrader T, Sagasti A, Bronstein JM (2016) Neurotoxicity of the Parkinson disease-associated pesticide ziram is synuclein-dependent in zebrafish embryos. Environ Health Perspect 124:1766-1775. 
Ma PM (2003) Catecholaminergic systems in the zebrafish. IV. Organization and projection pattern of dopaminergic neurons in the diencephalon. J Comp Neurol 460:13-37.

Matsuda W, Furuta T, Nakamura KC, Hioki H, Fujiyama F, Arai R, Kaneko $\mathrm{T}$ (2009) Single nigrostriatal dopaminergic neurons form widely spread and highly dense axonal arborizations in the neostriatum. J Neurosci 29:444-453.

Milescu LS, Bean BP, Smith JC (2010) Isolation of somatic Na+ currents by selective inactivation of axonal channels with a voltage prepulse. J Neurosci 30:7740-7748.

Neuhoff H, Neu A, Liss B, Roeper J (2002) I-h channels contribute to the different functional properties of identified dopaminergic subpopulations in the midbrain. J Neurosci 22:1290-1302.

O'Donnell KC, Lulla A, Stahl MC, Wheat ND, Bronstein JM, Sagasti A (2014) Axon degeneration and PGC- $1 \alpha$-mediated protection in a zebrafish model of $\alpha$-synuclein toxicity. Dis Model Mech 7:571-582.

Pape HC (1996) Queer current and pacemaker: the hyperpolarization-activated cation current in neurons. Annu Rev Physiol 58:299-327.

Ping HX, Shepard PD (1996) Apamin-sensitive $\mathrm{Ca}(2+)$-activated K+ channels regulate pacemaker activity in nigral dopamine neurons. Neuroreport 7:809-814.

Puopolo M, Raviola E, Bean BP (2007) Roles of subthreshold calcium current and sodium current in spontaneous firing of mouse midbrain dopamine neurons. J Neurosci 27:645-656.

Putzier I, Kullmann PHM, Horn JP, Levitan ES (2009) Ca(v)1.3 channel voltage dependence, not $\mathrm{Ca} 2+$ selectivity, drives pacemaker activity and amplifies bursts in nigral dopamine neurons. J Neurosci 29:15414-15419.

Reinig S, Driever W, Arrenberg AB (2017) The descending diencephalic dopamine system is tuned to sensory stimuli. Curr Biol 27:318-333.

Richards CD, Shiroyama T, Kitai ST (1997) Electrophysiological and immunocytochemical characterization of GABA and dopamine neurons in the substantia nigra of the rat. Neuroscience 80:545-557.

Rink E, Wullimann MF (2001) The teleostean (zebrafish) dopaminergic system ascending to the subpallium (striatum) is located in the basal diencephalon (posterior tuberculum). Brain Res 889:316-330.

Rink E, Wullimann MF (2002) Development of the catecholaminergic system in the early zebrafish brain: an immunohistochemical study. Brain Res Dev Brain Res 137:89-100.

Simuni T (2020) Parkinson study group STEADY-PD III investigators: isradipine versus placebo in early Parkinson disease: a randomized trial. Ann Intern Med 172:591-598.

Soman S, Keatinge M, Moein M, Da Costa M, Mortiboys H, Skupin A, Sugunan S, Bazala M, Kuznicki J, Bandmann O (2017) Inhibition of the mitochondrial calcium uniporter rescues dopaminergic neurons in pink1-/- zebrafish. Eur J Neurosci 45:528-535.

Surmeier DJ (2018) Determinants of dopaminergic neuron loss in Parkinson's disease. FEBS J 285:3657-3668.

Surmeier DJ, Mercer JN, Chan CS (2005) Autonomous pacemakers in the basal ganglia: who needs excitatory synapses anyway? Curr Opin Neurobiol 15:312-318.
Talley EM, Solorzano G, Lei QB, Kim D, Bayliss DA (2001) CNS distribution of members of the two-pore-domain (KCNK) potassium channel family. J Neurosci 21:7491-7505.

Tay TL, Ronneberger O, Ryu S, Nitschke R, Driever W (2011) Comprehensive catecholaminergic projectome analysis reveals singleneuron integration of zebrafish ascending and descending dopaminergic systems. Nat Commun 2:171.

Van Laar VS, Dukes AA, Cascio M, Hastings TG (2008) Proteomic analysis of rat brain mitochondria following exposure to dopamine quinone: implications for Parkinson disease. Neurobiol Dis 29:477-489.

Van Laar VS, Chen J, Zharikov AD, Bai Q, Di Maio R, Dukes AA, Hastings TG, Watkins SC, Greenamyre JT, St Croix CM, Burton EA (2020) $\alpha$-Synuclein amplifies cytoplasmic peroxide flux and oxidative stress provoked by mitochondrial inhibitors in CNS dopaminergic neurons in vivo. Redox Biol 37:101695.

Watson L, Keatinge M, Gegg M, Bai Q, Sandulescu MC, Vardi A, Futerman AH, Schapira AHV, Burton EA, Bandmann O (2019) Ablation of the pro-inflammatory master regulator miR-155 does not mitigate neuroinflammation or neurodegeneration in a vertebrate model of Gaucher's disease. Neurobiol Dis 127:563-569.

Wen L, Wei W, Gu W, Huang P, Ren X, Zhang Z, Zhu Z, Lin S, Zhang B (2008) Visualization of monoaminergic neurons and neurotoxicity of MPTP in live transgenic zebrafish. Dev Biol 314:84-92.

Wolfart J, Roeper J (2002) Selective coupling of T-type calcium channels to SK potassium channels prevents intrinsic bursting in dopaminergic midbrain neurons. J Neurosci 22:3404-3413.

Wolfart J, Neuhoff H, Franz O, Roeper J (2001) Differential expression of the small-conductance, calcium-activated potassium channel SK3 is critical for pacemaker control in dopaminergic midbrain neurons. J Neurosci 21:3443-3456.

Wullimann MF (2014) Ancestry of basal ganglia circuits: new evidence in teleosts. J Comp Neurol 522:2013-2018.

Xie W, Jiao B, Bai Q, Ilin VA, Sun M, Burton CE, Kolodieznyi D, Calderon MJ, Stolz DB, Opresko PL, St Croix CM, Watkins S, Van Houten B, Bruchez MP, Burton EA (2020) Chemoptogenetic ablation of neuronal mitochondria in vivo with spatiotemporal precision and controllable severity. Elife 9:e51845

Yamamoto K, Ruuskanen JO, Wullimann MF, Vernier P (2011) Differential expression of dopaminergic cell markers in the adult zebrafish forebrain. J Comp Neurol 519:576-598.

Yung WH, Häusser MA, Jack JJ (1991) Electrophysiology of dopaminergic and non-dopaminergic neurones of the guinea-pig substantia nigra pars compacta in vitro. J Physiol 436:643-667.

Zhang Y, Nguyen DT, Olzomer EM, Poon GP, Cole NJ, Puvanendran A, Phillips BR, Hesselson D (2017) Rescue of Pink1 deficiency by stress-dependent activation of autophagy. Cell Chem Biol 24:471-480.e4.

Zharikov AD, Cannon JR, Tapias V, Bai Q, Horowitz MP, Shah V, El Ayadi A, Hastings TG, Greenamyre JT, Burton EA (2015) shRNA targeting $\alpha$-synuclein prevents neurodegeneration in a Parkinson's disease model. J Clin Invest 125:2721-2735. 